\title{
On Minimal Log Discrepancies on Varieties with Fixed Gorenstein Index
}

\author{
YUSUKE NAKAMURA
}

\begin{abstract}
We generalize the rationality theorem of the accumulation points of log canonical thresholds, which was proved by Hacon, McKernan, and Xu. Further, we apply the rationality to the ACC problem on the minimal log discrepancies. We study the set of log discrepancies on varieties with fixed Gorenstein index. As a corollary, we prove that the minimal log discrepancies of three-dimensional canonical pairs with fixed coefficients satisfy the ACC.
\end{abstract}

\section{Introduction}

The minimal log discrepancy (mld for short) was introduced by Shokurov in order to reduce the conjecture of terminations of flips to a local problem about singularities. Recently, this has been a fundamental invariant in the minimal model program. There are two conjectures on mlds, the ACC (ascending chain condition) conjecture and the LSC (lower semicontinuity) conjecture. Shokurov [22] showed that these two conjectures imply the conjecture of terminations of flips.

In this paper, we consider the ACC conjecture. For an $\mathbb{R}$-divisor $D$ and a subset $I \subset \mathbb{R}$, we write $D \in I$ when all the nonzero coefficients of $D$ belong to $I$. Further, for a subset $I \subset \mathbb{R}$, we say that $I$ satisfies the ascending chain condition (resp. the descending chain condition) when there is no infinite increasing (resp. decreasing) sequence $a_{i} \in I ; A C C$ (resp. DCC) stands for the ascending chain condition (resp. the descending chain condition).

ConjeCture 1.1 (ACC conjecture [21, Conj. 4.2]). Fix $d \in \mathbb{Z}_{>0}$ and a subset $I \subset[0,1]$ that satisfies the DCC. Then the set

$$
A(d, I):=\left\{\operatorname{mld}_{x}(X, \Delta) \mid(X, \Delta) \text { is a log pair, } \operatorname{dim} X=d, \Delta \in I, x \in X\right\}
$$

satisfies the ACC, where $x$ is a closed point of $X$.

We are mainly interested in the case where $I$ is a finite set. This is because the ACC conjecture for an arbitrary finite set $I$ and the LSC conjecture imply the termination of flips [22].

The ACC conjecture is known for $d \leq 2$ by Alexeev [1] and Shokurov [20], and for toric pairs by Ambro [3]. Kawakita [11] proved the ACC conjecture on the interval [1,3] for three-dimensional smooth varieties. Further, Kawakita [10] proved that the ACC conjecture is true for a fixed variety $X$ and a finite set $I$.

Received January 6, 2015. Revision received February 13, 2015. 
More generally, he proved the discreteness of the set of log discrepancies for log triples (see Subsect. 2.1 for the definition)

$$
\left\{a_{E}(X, \Delta, \mathfrak{a}) \mid(X, \Delta, \mathfrak{a}) \text { is } \mathrm{lc}, \mathfrak{a} \in I, E \in \mathcal{D}_{X}\right\}
$$

when the pair $(X, \Delta)$ is fixed and $I$ is a finite set. Here, we denoted by $\mathcal{D}_{X}$ the set of all divisors over $X$. Further, $\mathfrak{a}=\prod \mathfrak{a}_{i}^{r_{i}}$ is an $\mathbb{R}$-ideal sheaf with coefficients $r_{i}$ in $I$. The purpose of this paper is to generalize this result to the family of the varieties with fixed Gorenstein index.

Theorem 1.2. Fix $d \in \mathbb{Z}_{>0}, r \in \mathbb{Z}_{>0}$, and a finite subset $I \subset[0,+\infty)$. Then the set

$$
B(d, r, I):=\left\{a_{E}(X, \mathfrak{a}) \mid(X, \mathfrak{a}) \in P(d, r), \mathfrak{a} \in I, E \in \mathcal{D}_{X}\right\} \subset[0,+\infty)
$$

is discrete in $\mathbb{R}$. Here we denote by $P(d, r)$ the set of all $d$-dimensional lc pairs $(X, \mathfrak{a})$ such that $r K_{X}$ is a Cartier divisor.

Since $\operatorname{mld}_{x}(X, \mathfrak{a})=a_{E}(X, \mathfrak{a})$ holds for some $E \in \mathcal{D}_{X}$, we get the following corollary.

Corollary 1.3. Fix $d \in \mathbb{Z}_{>0}, r \in \mathbb{Z}_{>0}$ and a finite subset $I \subset[0,+\infty)$. Then the following set

$$
A^{\prime}(d, r, I):=\left\{\operatorname{mld}_{x}(X, \mathfrak{a}) \mid X \in P(d, r), \mathfrak{a} \in I, x \in X\right\} \subset[0,+\infty)
$$

is discrete in $\mathbb{R}$. Here we denote by $P(d, r)$ the set of all $d$-dimensional lc pairs $(X, \mathfrak{a})$ such that $r K_{X}$ is a Cartier divisor.

Corollary 1.3 does not imply the finiteness of $A^{\prime}(d, r, I)$ because we do not know the boundedness of $A^{\prime}(d, r, I)$. Hence, Corollary 1.3 shows the finiteness of $A^{\prime}(d, r, I)$ modulo the BDD (boundedness) conjecture, which states the boundedness of minimal log discrepancies.

CONJECTURE 1.4 (BDD conjecture). For fixed $d \in \mathbb{Z}_{>0}$, there exists a real number $a(d)$ such that $\operatorname{mld}(X) \leq a(d)$ holds for any $\mathbb{Q}$-Gorenstein d-dimensional normal variety $X$.

The BDD conjecture is known only for $d \leq 3$ [18]. In arbitrary dimension, the conjecture is known for the set of varieties with bounded multiplicity [9].

As a corollary of Corollary 1.3, we can prove the ACC for three-dimensional canonical pairs.

COROLlary 1.5. If $I \subset[0,1]$ is a finite subset, the set

$\left\{\operatorname{mld}_{x}(X, \Delta) \mid(X, \Delta)\right.$ is a canonical pair, $\left.\operatorname{dim} X=3, \Delta \in I, x \in X\right\}$,

denoted by $A_{\mathrm{can}}(3, I)$, satisfies the ACC. Further, 1 is the only accumulation point of $A_{\mathrm{can}}(3, I)$. 
Theorem 1.2 is proved by induction on $\operatorname{dim}_{\mathbb{Q}} \operatorname{Span}_{\mathbb{Q}}(I \cup\{1\})$, the dimension of the $\mathbb{Q}$-vector space generated by $I \cup\{1\}$. In the inductive step, we need the following theorem about a perturbation of an irrational coefficient of log canonical pairs.

Theorem 1.6. Fix $d \in \mathbb{Z}_{>0}$. Let $r_{1}, \ldots, r_{c^{\prime}}$ be positive real numbers, and let $r_{0}=1$. Assume that $r_{0}, \ldots, r_{c^{\prime}}$ are $\mathbb{Q}$-linearly independent. Let $s_{1}, \ldots, s_{c}$ : $\mathbb{R}^{c^{\prime}+1} \rightarrow \mathbb{R}$ be $\mathbb{Q}$-linear functions from $\mathbb{R}^{c^{\prime}+1}$ to $\mathbb{R}$ (that is, the extensions of $\mathbb{Q}$-linear functions from $\mathbb{Q}^{c^{\prime}+1}$ to $\mathbb{Q}$ by taking the tensor product $\otimes_{\mathbb{Q}} \mathbb{R}$ ). Assume that $s_{i}\left(r_{0}, \ldots, r_{c^{\prime}}\right) \in \mathbb{R}_{\geq 0}$ for each $i$. Then there exists a positive real number $\epsilon>0$ such that the following holds: For any $\mathbb{Q}$-Gorenstein normal variety $X$ of dimension $d$ and $\mathbb{Q}$-Cartier effective Weil divisors $D_{1}, \ldots, D_{c}$ on $X$, if $\left(X, \sum_{1 \leq i \leq c} s_{i}\left(r_{0}, \ldots, r_{c^{\prime}}\right) D_{i}\right)$ is lc, then $\left(X, \sum_{1 \leq i \leq c} s_{i}\left(r_{0}, \ldots, r_{c^{\prime}-1}, t\right) D_{i}\right)$ is also lc for any $t$ satisfying $\left|t-r_{c^{\prime}}\right| \leq \epsilon$.

Remark 1.7. The positive real number $\epsilon$ in Theorem 1.6 does not depend on $X$, but depends only on $d, r_{1}, \ldots, r_{c^{\prime}}$, and $s_{1}, \ldots, s_{c}$.

Kawakita [10] proved this theorem for a fixed variety $X$ using a method of generic limit and proves the discreteness of $\log$ discrepancies for fixed $X$. When $c^{\prime}=1$ and each $s_{i}$ satisfies $s_{i}\left(\mathbb{R}_{\geq 0}^{2}\right) \subset \mathbb{R}_{\geq 0}$, this theorem just states the rationality of accumulation points of $\log$ canonical thresholds proved by Hacon, McKernan, and $\mathrm{Xu}[8, \mathrm{Thm} .1 .11]$. Actually, the proof of Theorem 1.6 heavily depends on their argument. We also note that the rationality of accumulation points of log canonical thresholds on smooth varieties was proved by Kollár [14, Thm. 7] and by de Fernex and Mustaţă [5, Cor. 1.4] using a method of generic limit.

The paper is organized as follows. In Section 2, we review some definitions and facts from the minimal model theory. Further, we list some results on the ACC for log canonical thresholds by Hacon, McKernan, and Xu [8]. In Section 3, we prove the key proposition (Theorem 3.8), which is necessary to prove Theorem 1.6. The essential idea of proof is due to the paper [8]. In Section 4, we prove Theorem 1.6. In Section 5, we prove the main theorem (Theorem 1.2) and the corollaries.

\subsection{Notation and Convention}

Throughout this paper, we work over the field of complex numbers $\mathbb{C}$.

- For an $\mathbb{R}$-divisor $D$ and a subset $I \subset \mathbb{R}$, we write $D \in I$ when all the nonzero coefficients of $D$ belong to $I$.

- For an $\mathbb{R}$-ideal sheaf $\mathfrak{A}=\prod \mathfrak{a}_{i}^{r_{i}}$ and a subset $I \subset \mathbb{R}$, we write $\mathfrak{A} \in I$ when all the nonzero coefficients $r_{i}$ of $\mathfrak{A}$ belong to $I$.

\section{Preliminaries}

\subsection{Minimal Log Discrepancies}

We recall some notation in the theory of singularities in the minimal model program. For more details, we refer the reader to [15]. 
A log pair $(X, \Delta)$ is a normal variety $X$ and an effective $\mathbb{R}$-divisor $\Delta$ such that $K_{X}+\Delta$ is $\mathbb{R}$-Cartier. If $X$ is $\mathbb{Q}$-Gorenstein, we sometimes identify $X$ with the log pair $(X, 0)$.

An $\mathbb{R}$-ideal sheaf on $X$ is a formal product $\mathfrak{a}_{1}^{r_{1}} \cdots \mathfrak{a}_{s}^{r_{s}}$, where $\mathfrak{a}_{1}, \ldots, \mathfrak{a}_{s}$ are ideal sheaves on $X$, and $r_{1}, \ldots, r_{s}$ are positive real numbers. For a $\log$ pair $(X, \Delta)$ and an $\mathbb{R}$-ideal sheaf $\mathfrak{a}$, we call $(X, \Delta, \mathfrak{a})$ a $\log$ triple. When $\Delta=0$ (resp. $\mathfrak{A}=$ $\left.\mathcal{O}_{X}\right)$, we sometimes drop $\Delta(\operatorname{resp} . \mathfrak{A})$ and write $(X, \mathfrak{a})(\operatorname{resp} .(X, \Delta))$.

For a proper birational morphism $f: X^{\prime} \rightarrow X$ from a normal variety $X^{\prime}$ and a prime divisor $E$ on $X^{\prime}$, the $\log$ discrepancy of $(X, \Delta, \mathfrak{a})$ at $E$ is defined as

$$
a_{E}(X, \Delta, \mathfrak{a}):=1+\operatorname{coeff}_{E}\left(K_{X^{\prime}}-f^{*}\left(K_{X}+\Delta\right)\right)-\operatorname{ord}_{E} \mathfrak{a},
$$

where $\operatorname{ord}_{E} \mathfrak{a}:=\sum_{i=1}^{s} r_{i} \operatorname{ord}_{E} \mathfrak{a}_{i}$. The image $f(E)$ is called the center of $E$ on $X$, and we denote it by $\mathrm{c}_{X}(E)$. For a closed subset $Z$ of $X$, the minimal $\log$ discrepancy ( $m l d$ for short) over $Z$ is defined as

$$
\operatorname{mld}_{Z}(X, \Delta, \mathfrak{a}):=\inf _{\mathrm{c}_{X}(E) \subset Z} a_{E}(X, \Delta, \mathfrak{a}) .
$$

In this definition, the infimum is taken over all prime divisors $E$ on $X^{\prime}$ with center $\mathrm{c}_{X}(E) \subset Z$, where $X^{\prime}$ is a higher birational model of $X$, that is, $X^{\prime}$ is the source of some proper birational morphism $X^{\prime} \rightarrow X$.

REMARK 2.1. It is known that $\operatorname{mld}_{Z}(X, \Delta, \mathfrak{a})$ is in $\mathbb{R}_{\geq 0} \cup\{-\infty\}$ and that if $\operatorname{mld}_{Z}(X, \Delta, \mathfrak{a}) \geq 0$, then the infimum on the right-hand side in the definition is, in fact, the minimum.

Remark 2.2. Let $D_{i}$ be effective Weil divisors on $X$, and $\mathfrak{a}_{i}:=\mathcal{O}_{X}\left(-D_{i}\right)$ the corresponding ideal sheaves. When $X$ is $\mathbb{Q}$-Gorenstein and $D_{i}$ are Cartier divisors, we can identify $\left(X, \sum r_{i} D_{i}\right)$ and $\left(X, \prod \mathfrak{a}_{i}^{r_{i}}\right)$. Indeed, for any divisor $E$ over $X$, we have $a_{E}\left(X, \sum r_{i} D_{i}\right)=a_{E}\left(X, \prod \mathfrak{a}_{i}^{r_{i}}\right)$.

For simplicity of notation, we write $\operatorname{mld}_{x}(X, \Delta, \mathfrak{a})$ instead of $\operatorname{mld}_{\{x\}}(X, \Delta, \mathfrak{a})$ for a closed point $x$ of $X$ and write $\operatorname{mld}(X, \Delta, \mathfrak{a})$ instead of $\operatorname{mld}_{X}(X, \Delta, \mathfrak{a})$.

We say that the pair $(X, \Delta, \mathfrak{a})$ is $\log$ canonical (lc for short) if $\operatorname{mld}(X, \Delta, \mathfrak{a}) \geq$ 0 . Further, we say that the pair $(X, \Delta, \mathfrak{a})$ is Kawamata log terminal (klt for short) if $\operatorname{mld}(X, \Delta, \mathfrak{a})>0$. When $E$ is a divisor over $X$ such that $a_{E}(X, \Delta, \mathfrak{a}) \leq 0$, the center $\mathrm{c}_{X}(E)$ is called a non-klt center.

We say that the pair $(X, \Delta, \mathfrak{a})$ is canonical (resp. terminal) if $a_{E}(X, \Delta, \mathfrak{a}) \geq 1$ (resp. $>1$ ) for any exceptional divisor $E$ over $X$.

\subsection{Extraction of Divisors}

In this subsection, we recall some known results on extractions of divisors.

We can extract a divisor whose log discrepancy is at most one.

TheOREM 2.3. Let $(X, \Delta)$ be a klt pair, and let $E$ be a divisor over $X$ such that $a_{E}(X, \Delta) \leq 1$. Then there exists a projective birational morphism $\pi: Y \rightarrow X$ such that $Y$ is $\mathbb{Q}$-factorial and the only exceptional divisor is $E$. 
Proof. This is a particular case of [4, Cor. 1.4.3].

When $(X, \Delta)$ is lc, we can find a modification which is dit. We call a log pair $(X, \Delta)$ divisorial log terminal (dlt for short) when there exists a log resolution $f: Y \rightarrow X$ such that $a_{E}(X, \Delta)>0$ for any $f$-exceptional divisor $E$ on $Y$.

THeORem 2.4 (dlt modification). Let $(X, \Delta)$ be an lc pair. Then there exists a projective birational morphism $f: Y \rightarrow X$ with the following properties:

- $Y$ is $\mathbb{Q}$-factorial.

- $\left(Y, \Delta_{Y}\right)$ is dlt, where we define $\Delta_{Y}$ as $K_{Y}+\Delta_{Y}=f^{*}\left(K_{X}+\Delta\right)$.

- $a_{E}(X, \Delta)=0$ for every $f$-exceptional divisor $E$.

Proof. See, for instance, [6, Thm. 10.4].

\subsection{ACC for Log Canonical Thresholds}

In Section 3, we need the following ACC properties proved by Hacon, McKernan, and $\mathrm{Xu}[8]$.

Theorem 2.5 (Hacon, McKernan, Xu [8, Thm. 1.4]). Fix $d \in \mathbb{Z}_{>0}$ and a subset $I \subset[0,1]$ satisfying the $D C C$.

Then there is a finite subset $I_{0} \subset I$ with the following property: If $(X, \Delta)$ is a log pair such that:

- $(X, \Delta)$ is $l c, \operatorname{dim} X=d, \Delta \in I$, and

- there exists a non-klt center $Z \subset X$ which is contained in every component of $\Delta$,

then $\Delta \in I_{0}$.

Theorem 2.6 (Hacon, McKernan, Xu [8, Thm. 1.5]). Fix $d \in \mathbb{Z}_{>0}$ and a subset $I \subset[0,1]$ satisfying the DCC.

Then there is a finite subset $I_{0} \subset I$ with the following property: If $(X, \Delta)$ is a projective log pair such that:

- $(X, \Delta)$ is $l c, \operatorname{dim} X=d, \Delta \in I$, and

- $K_{X}+\Delta \equiv 0$,

then $\Delta \in I_{0}$.

\section{Accumulation Points of Log Canonical Thresholds}

The goal of this section is to prove Corollary 3.9. It is a generalization of [8, Thm. 1.11] and necessary for the proof of Theorem 1.6.

Usually, the log canonical threshold is defined as follows: for an lc pair $(X, \Delta)$ and a $\mathbb{Q}$-Cartier $\mathbb{Z}$-Weil effective divisor $M$,

$$
\operatorname{LCT}(\Delta ; M):=\sup \left\{c \in \mathbb{R}_{\geq 0} \mid(X, \Delta+c M) \text { is lc }\right\} .
$$

However, for the proof of Theorem 1.6, we need to treat the case where $M$ is not effective. According to this reason, we introduce the new threshold set $\mathfrak{L}_{d}(I)$. It 
no longer satisfies the ACC, but we can prove the rationality of the accumulation points (Cor. 3.9).

Corollary 3.9 easily follows from Theorem 3.6 and Theorem 3.8. They are proved in essentially the same way of the proof of Proposition 11.5 and Proposition 11.7 in [8]. For the reader's convenience, we follow the proof of Proposition 11.5 and Proposition 11.7 in [8] and use as same notation as possible.

First, we introduce some notation. For a subset $I \subset[0,+\infty)$, we define

$$
I_{+}:=\{0\} \cup\left\{\sum_{1 \leq i \leq l} r_{i} \mid l \in \mathbb{Z}_{>0}, r_{1}, \ldots, r_{l} \in I\right\} .
$$

This becomes a discrete set if $I$ is discrete. When $D_{i}$ are finitely many distinct prime divisors and $d_{i}(t): \mathbb{R} \rightarrow \mathbb{R}$ are affine functions, we call the formal finite sum $\sum_{i} d_{i}(t) D_{i}$ an affine functional divisor.

Definition $3.1\left(\mathcal{D}_{c}(I)\right)$. Fix $c \in \mathbb{R}_{\geq 0}$ and a subset $I \subset[0,+\infty)$. For an affine functional divisor $\Delta(t)=\sum_{i} d_{i}(t) D_{i}$, we write $\Delta(t) \in \mathcal{D}_{c}(I)$ when the following conditions are satisfied:

- Each $d_{i}(t)$ is equal to 1 or of the form $\frac{m-1+f+k t}{m}$, where $m \in \mathbb{Z}_{>0}, f \in I_{+}$, and $k \in \mathbb{Z}$.

- Further, $f+k t$ above can be written as $f+k t=\sum_{j}\left(f_{j}+k_{j} t\right)$, where $f_{j} \in$ $I \cup\{0\}, k_{j} \in \mathbb{Z}$, and $f_{j}+k_{j} c \geq 0$ for each $j$.

In such a case, by abuse of notation, we also write $d_{i}(t) \in \mathcal{D}_{c}(I)$.

The form of the coefficient $d_{i}(t)$ is preserved by adjunction.

Lemma 3.2. Fix $c \in \mathbb{R}_{\geq 0}$ and a subset $I \subset[0,1]$. Let $X$ be a $\mathbb{Q}$-factorial normal variety, and $\Delta(t)=\sum_{0 \leq i \leq c} d_{i}(t) D_{i}$ be an affine functional divisor on $X$. Assume the following conditions:

- $\Delta(t) \in \mathcal{D}_{c}(I)$, and $(X, \Delta(c))$ is $l c$.

- $d_{0}(t)$ is identically one, and $d_{i}(c)>0$ for each $i$.

Let $S^{\mathrm{n}}$ be the normalization of $S:=D_{0}$. Define the affine functional divisor $\Delta_{S^{\mathrm{n}}}(t)$ on $S^{\mathrm{n}}$ by adjunction:

$$
\left.\left(K_{X}+\Delta(t)\right)\right|_{S^{\mathrm{n}}}=K_{S^{\mathrm{n}}}+\Delta_{S^{\mathrm{n}}}(t) .
$$

Then $\Delta_{S^{\mathrm{n}}}(t) \in \mathcal{D}_{c}(I)$.

Proof. The statement follows from [16, Prop. 16.6]. We give a sketch of a proof.

Let $p \in S$ be a codimension one point of $S$.

Suppose that $\left(X, D_{0}\right)$ is not plt at $p$. Then $p \notin \operatorname{Supp} D_{i}$ for any $i \geq 1$, and $\operatorname{coeff}_{p} \operatorname{Diff}_{S^{\mathrm{n}}}(0)=0$ or 1 [16, Prop. 16.6.1-2]. Hence, we have coeff $p \Delta_{S^{\mathrm{n}}}(t)=0$ or 1 for any $t$.

Suppose that $\left(X, D_{0}\right)$ is plt at $p$. Then coeff $_{p} \operatorname{Diff}_{S^{n}}(0)=\frac{m-1}{m}$ for some $m \in$ $\mathbb{Z}_{>0}$, and $m D$ becomes Cartier at $p$ for any Weil divisor $D$ [16, Prop. 16.6.3]. 
Hence, $\operatorname{coeff}_{p} \Delta_{S^{\mathrm{n}}}(t)$ is of the form

$$
\frac{m-1}{m}+\frac{1}{m} \sum_{j} \frac{n_{j}-1+f_{j}+k_{j} t}{n_{j}},
$$

where $\left(n_{j}-1+f_{j}+k_{j} t\right) / n_{j}$ is of the form as in the definition of $\mathcal{D}_{c}(I)$. We can prove that such a form also satisfies the condition in the definition of $\mathcal{D}_{c}(I)$ by easy calculation (cf. [19, Lemma 4.4]).

We define $\mathfrak{L}_{d}(I)$, the set of all $\log$ canonical thresholds derived from coefficients $I$.

Definition $3.3\left(\mathfrak{L}_{d}(I)\right)$. Let $d \in \mathbb{Z}_{>0}$, and let $I \subset[0,+\infty)$ be a subset. We define $\mathfrak{L}_{d}(I) \subset \mathbb{R}_{\geq 0}$ as follows: $c \in \mathfrak{L}_{d}(I)$ if and only if there exist a $\mathbb{Q}$-Gorenstein normal varieties $X$ and an affine functional divisor $\Delta(t)$ with the following conditions:

- $\operatorname{dim} X \leq d, \Delta(t) \in \mathcal{D}_{c}(I)$,

- $\Delta(a)$ is $\mathbb{R}$-Cartier for any $a \in \mathbb{R}$,

- $(X, \Delta(c))$ is lc, and

- $(X, \Delta(c+\epsilon))$ is not lc for any $\epsilon>0$, or $(X, \Delta(c-\epsilon))$ is not lc for any $\epsilon>0$.

REMARK 3.4. When we say that $(X, \Delta)$ is an lc pair, we assume that $\Delta$ is effective. Therefore, we say that $(X, \Delta)$ is not lc when $\Delta$ is not effective.

Further, we define $\mathfrak{G}_{d}(I)$, the set of all numerically trivial thresholds derived from coefficients $I$.

Definition $3.5\left(\mathfrak{G}_{d}(I)\right)$. Let $d \in \mathbb{Z}_{>0}$, and let $I \subset[0,+\infty)$ be a subset. We define $\mathfrak{G}_{d}(I) \subset \mathbb{R}_{\geq 0}$ as follows: $c \in \mathfrak{G}_{d}(I)$ if and only if there exist a $\mathbb{Q}$-factorial normal projective variety $X$ and an affine functional divisor $\Delta(t)$ with the following conditions:

- $\operatorname{dim} X \leq d, \Delta(t) \in \mathcal{D}_{c}(I)$,

- $(X, \Delta(c))$ is lc, and $K_{X}+\Delta(c) \equiv 0$.

- $K_{X}+\Delta\left(c^{\prime}\right) \not \equiv 0$ for some $c^{\prime} \neq c$ (equivalently, for all $c^{\prime} \neq c$ ).

By the following theorem, we can reduce a local problem to a global problem.

TheOREM 3.6. Let $d \geq 2$, and let $I \subset[0,+\infty)$ be a subset. Then $\mathfrak{L}_{d}(I) \subset$ $\mathfrak{G}_{d-1}(I)$.

Lemma 3.7. Let $c \in \mathbb{R}_{\geq 0}$, and let $I \subset[0,+\infty)$ be a subset. Suppose that there exists an affine function $d(t): \mathbb{R} \rightarrow \mathbb{R}$ with the following conditions:

- $d(t) \in \mathcal{D}_{c}(I)$, and $d(t)$ is not a constant function.

- $d(c)=0$ or 1 .

Then $c \in \mathfrak{G}_{d}(I)$ for any $d \geq 1$. Especially, $\frac{f}{k} \in \mathfrak{G}_{d}(I)$ for any $d \geq 1, f \in I \cup\{0\}$, and $k \in \mathbb{Z}_{>0}$.

Proof. We can easily construct on a curve. 
Proof of Theorem 3.6. Let $c \in \mathfrak{L}_{d}(I)$, and let $(X, \Delta(t))$ be as in Definition 3.3. Assume that $(X, \Delta(c+\epsilon))$ is not lc for any $\epsilon>0$ (the same proof works in the other case). We may write $\Delta(t)=\sum_{i} d_{i}(t) D_{i}$ with distinct prime divisors $D_{i}$. By Lemma 3.7 we may assume that $d_{i}(c)>0$ for any $i$. Then $\Delta(c+\epsilon) \geq 0$ for sufficiently small $\epsilon>0$.

Let $f: Y \rightarrow X$ be a dlt modification (Theorem 2.4) of $(X, \Delta(c))$. Then $Y$ is $\mathbb{Q}$-factorial, and we can write

$$
K_{Y}+T+\Delta^{\prime}(c)=f^{*}\left(K_{X}+\Delta(c)\right)
$$

where $\Delta^{\prime}(t)$ is the strict transform of $\Delta(t)$, and $T$ is the sum of the exceptional divisors. Since the pair $\left(Y, T+\Delta^{\prime}(c)\right)$ is dlt, there exists a divisor $E$ on $Y$ such that

$$
a_{E}(X, \Delta(c))=0 \quad \text { and } \quad a_{E}(X, \Delta(c+\epsilon))<0
$$

for any $\epsilon>0$. If $E$ is not $f$-exceptional, then $d_{i}(c)=1$ for some $d_{i}(t)$ that is not identically one. In this case, $c \in \mathfrak{G}_{d-1}(I)$ by Lemma 3.7.

In what follows, we assume that $E$ is $f$-exceptional and so a component of Supp $T$. By adjunction, we can define an affine functional divisor $\Delta_{E}(t)$ on $E$ such that

$$
\left.\left(K_{Y}+T+\Delta^{\prime}(t)\right)\right|_{E}=K_{E}+\Delta_{E}(t) .
$$

Here, $\Delta_{E}(t) \in \mathcal{D}_{c}(I)$ by Lemma 3.2.

Let $F$ be a general fiber of $E \rightarrow f(E)$. Define $\Delta_{F}(t)$ as

$$
\left.\left(K_{E}+\Delta_{E}(t)\right)\right|_{F}=K_{F}+\Delta_{F}(t) .
$$

Then $\left(F, \Delta_{F}(t)\right)$ satisfies:

- $\operatorname{dim} F \leq d-1, F$ is projective,

- $\Delta_{F}(t) \in \mathcal{D}_{c}(I)$,

- $K_{F}+\Delta_{F}(c)=\left.f^{*}\left(K_{X}+\Delta(c)\right)\right|_{F} \equiv 0$, and

- $\left(F, \Delta_{F}(c)\right)$ is lc.

Hence, $\left(F, \Delta_{F}(t)\right)$ satisfies all conditions in Definition 3.5 except for $K_{F}+$ $\Delta_{F}\left(c^{\prime}\right) \not \equiv 0$ for some $c^{\prime}$.

We may write $\Delta(t)=\Delta+t M$ with an $\mathbb{R}$-divisor $\Delta$ and a $\mathbb{Q}$-divisor $M$. Write $M=M_{+}-M_{-}$, where $M_{+} \geq 0$ and $M_{-} \geq 0$ have no common components. Since $a_{E}(X, \Delta+(c+\epsilon) M)<a_{E}(X, \Delta+c M)=0$, it follows that $\operatorname{ord}_{E} M_{+}>\operatorname{ord}_{E} M_{-} \geq 0$. Possibly replacing $E$ by other component of $T$, we may assume that

$$
\operatorname{ord}_{E} M_{-} \cdot \operatorname{ord}_{E_{j}} M_{+} \leq \operatorname{ord}_{E_{j}} M_{-} \cdot \operatorname{ord}_{E} M_{+}
$$

for any component $E_{j} \subset \operatorname{Supp} T$. We may take $\epsilon_{1} \geq \epsilon_{2}>0$ such that $a_{E}(X, \Delta+$ $\left.\left(c+\epsilon_{1}\right) M-\epsilon_{2} M_{+}\right)=0$. Note that

$$
\epsilon_{1}\left(\operatorname{ord}_{E} M_{+}-\operatorname{ord}_{E} M_{-}\right)=\epsilon_{2} \operatorname{ord}_{E} M_{+} .
$$


Then we have

$$
\begin{aligned}
0 & \left.\equiv f^{*}\left(K_{X}+\Delta+\left(c+\epsilon_{1}\right) M-\epsilon_{2} M_{+}\right)\right|_{F} \\
& =\left.\left(K_{Y}+T+U+\Delta^{\prime}+\left(c+\epsilon_{1}\right) M^{\prime}-\epsilon_{2} M_{+}^{\prime}\right)\right|_{F} \\
& =K_{F}+\Delta_{F}\left(c+\epsilon_{1}\right)+\left.U\right|_{F}-\left.\epsilon_{2} M_{+}^{\prime}\right|_{F},
\end{aligned}
$$

where we set

$$
U=\sum_{j}\left(\epsilon_{1} \operatorname{ord}_{E_{j}} M-\epsilon_{2} \operatorname{ord}_{E_{j}} M_{+}\right) E_{j}
$$

Note that

$$
\begin{aligned}
& \epsilon_{1} \operatorname{ord}_{E_{j}} M-\epsilon_{2} \operatorname{ord}_{E_{j}} M_{+} \\
& \quad=\frac{\epsilon_{1}}{\operatorname{ord}_{E} M_{+}}\left(\operatorname{ord}_{E} M_{-} \cdot \operatorname{ord}_{E_{j}} M_{+}-\operatorname{ord}_{E_{j}} M_{-} \cdot \operatorname{ord}_{E} M_{+}\right) \\
& \quad \leq 0 .
\end{aligned}
$$

Therefore, $K_{F}+\left.\Delta_{F}\left(c+\epsilon_{1}\right) \equiv \epsilon_{2} M_{+}^{\prime}\right|_{F}-\left.U\right|_{F} \geq\left.\epsilon_{2} M_{+}^{\prime}\right|_{F}$. Since ord $M_{+}>0$, it follows that $f(E) \subset \operatorname{Supp} M_{+}$, and so $\left.M_{+}^{\prime}\right|_{F}>0$. Therefore, $K_{F}+\Delta_{F}\left(c+\epsilon_{1}\right)$ is not numerically trivial.

Theorem 3.8. Let $d \geq 2$, and let $I \subset[0,+\infty)$ be a finite subset. The accumulation points of $\mathfrak{G}_{d}(I)$ are contained in $\mathfrak{G}_{d-1}(I)$.

As a corollary, we can prove the rationality of the accumulation points of $\mathfrak{L}_{d}(I)$.

Corollary 3.9. Let $d \in \mathbb{Z}_{>0}$, and let $I \subset[0,+\infty)$ be a finite subset. The accumulation points of $\mathfrak{L}_{d}(I)$ are contained in $\operatorname{Span}_{\mathbb{Q}}(I \cup\{1\})$, where we denote by $\operatorname{Span}_{\mathbb{Q}}(I \cup\{1\}) \subset \mathbb{R}$ the $\mathbb{Q}$-vector space spanned by the elements of I and 1 .

We prove a stronger statement (cf. [8, Prop. 11.7]).

Proposition 3.10. Let $d \geq 2$, and let $I \subset[0,+\infty)$ be a finite subset. Further, let $c \in \mathbb{R}_{\geq 0}$.

Suppose that for each $i \in \mathbb{Z}_{>0}$, there exist $c_{i} \in \mathbb{R}_{\geq 0}, a \mathbb{Q}$-factorial normal projective variety $X_{i}$, and an affine functional divisor $\Delta_{i}(t)$ on $X_{i}$ with the following conditions:

- The sequence $c_{i}$ is increasing or decreasing. Further, $c_{i}$ is accumulating to $c$.

- $\operatorname{dim} X_{i} \leq d$ for each $i$.

- $\Delta_{i}(t)$ can be written as $\Delta_{i}(t)=A_{i}+B_{i}(t)$, where the coefficients of $A_{i}$ are approaching one, and $B_{i}(t) \in \mathcal{D}_{c_{i}}(I)$.

- $\left(X_{i}, \Delta_{i}\left(c_{i}\right)\right)$ is $l c$, and $K_{X_{i}}+\Delta_{i}\left(c_{i}\right) \equiv 0$.

- $K_{X_{i}}+\Delta_{i}\left(c_{i}^{\prime}\right) \not \equiv 0$ for some $c_{i}^{\prime} \neq c_{i}$.

Then $c \in \mathfrak{G}_{d-1}(I)$.

REMARK 3.11. If $c_{i} \in \mathfrak{G}_{d}(I)$, then $c_{i}$ satisfies the conditions of the proposition (in this case, $A_{i}=0$ ). Hence, Theorem 3.8 follows from Proposition 3.10. 
In the proof of Proposition 3.10, we reduce to the case where $X_{i}$ has Picard number one and apply the following lemma from [8].

Lemma 3.12 ([8, Lemma 11.6]). Let $(X, \Delta)$ be a projective $\mathbb{Q}$-factorial lc pair of dimension $d$ and of Picard number one. Assume that $K_{X}+\Delta \equiv 0$. If the coefficients of $\Delta$ are at least $\delta>0$, then $\Delta$ has at most $\frac{d+1}{\delta}$ components.

Proof of Proposition 3.10. Possibly replacing $A_{i}$ and $B_{i}(t)$, we may assume that every coefficient of $B_{i}(t)$ is not identically one. We may write $B_{i}(t)=$ $\sum_{l} d_{i l}(t) D_{i l}$ as in Definition 3.1.

By Lemma 3.7 we may assume that $(I \cup\{0\}) \cap c \mathbb{Z}_{>0}=\emptyset$. Then we may assume the following conditions on $B_{i}(t)$.

LEMMA 3.13. We may assume the following conditions:

(1) If $d_{i l}(t)=\frac{m-1+f+k t}{m}$ as in Definition 3.1, then $f$ and $k$ have only finitely many possibilities.

(2) $d_{i l}\left(c_{i}\right)$ are bounded from zero, and $d_{i l}\left(c_{i}\right)<1$ for any $i, l$.

(3) $d_{i l}(c)>0$.

(4) The set $\left\{d_{i l}(c) \mid i, l\right\}$ satisfies the DCC.

Proof. Since $(I \cup\{0\}) \cap c \mathbb{Z}_{>0}=\emptyset$, possibly passing to a tail of the sequence, we may assume that there exist $k^{\prime} \in \mathbb{Z}_{>0}$ and $\in \in \mathbb{R}_{>0}$ such that for any $f_{j} \in I \cup\{0\}$, $k_{j} \in \mathbb{Z}$, and $i$,

- $f_{j}+k_{j} c_{i} \geq 0$ implies $f_{j}+k_{j} c_{i} \geq \epsilon$ and $k_{j} \geq-k^{\prime}$ unless $f_{j}=k_{j}=0$.

Here, we note that $I$ is a finite set.

Let $d_{i l}(t)=\frac{m-1+f+k t}{m}$ be a coefficient of $B_{i}(t)$. By assumption, $f+k t$ can be written as $f+k t=\sum_{j}\left(f_{j}+k_{j} t\right)$, where $f_{j} \in I, k_{j} \in \mathbb{Z}$, and $f_{j}+k_{j} c_{i} \geq 0$ for each $j$.

Note that $f+k c_{i} \leq 1$ by the $\log$ canonicity. Since $f_{j}+k_{j} c_{i} \geq 0$ implies $f_{j}+$ $k_{j} c_{i} \geq \epsilon$ and $k_{j} \geq-k^{\prime}$, it follows that $k$ is bounded from below. Since $c_{i} \geq \epsilon$, it follows that $k$ is also bounded from above. Since the set $I_{+}$is discrete, $f$ has also only finitely many possibilities. Therefore, (1) follows.

By (1) it follows that $d_{i l}\left(c_{i}\right) \geq \min \left\{\frac{1}{2}, \epsilon\right\}$. Hence, $d_{i l}\left(c_{i}\right)$ are bounded from zero. Since $c_{i}$ are distinct, by (1), possibly passing to a subsequence, we may assume that $d_{i l}\left(c_{i}\right) \neq 1$ (and hence $d_{i l}\left(c_{i}\right)<1$ ) for any $i, l$. Thus, (2) follows.

(3) follows from (2), and (4) follows from (1).

By Lemma 3.13(2), possibly passing to a tail of the sequence, we may assume that $A_{i}$ and $B_{i}(t)$ have no common components and that $\left\lfloor A_{i}\right\rfloor=\left\lfloor A_{i}+B_{i}\left(c_{i}\right)\right\rfloor$.

In our setting, the following claim is important and allows the same argument as in [8] to work.

Claim 3.14. We may assume that $\left(X_{i},\left\lceil A_{i}\right\rceil+B_{i}(c)\right)$ is lc for any $i$. 
Proof. We may write $\Delta_{i}(t)=A_{i}+M_{i}+t\left(N_{i}^{+}-N_{i}^{-}\right)$, where $N_{i}^{+} \geq 0$ and $N_{i}^{-} \geq 0$ have no common components; $\left(X_{i}, A_{i}+M_{i}+c_{i}\left(N_{i}^{+}-N_{i}^{-}\right)\right)$is lc by the assumption.

First, suppose $c_{i}<c$. Note that $A_{i}+M_{i}+c_{i} N_{i}^{+}-c N_{i}^{-} \geq 0$ (Lemma 3.13(3)). Hence, $\left(X_{i}, A_{i}+M_{i}+c_{i} N_{i}^{+}-c N_{i}^{-}\right)$is also lc. Here, by Lemma 3.13(4), the coefficients of $M_{i}-c N_{i}^{-}$satisfy the DCC (note that a coefficient of $M_{i}-c N_{i}^{-}$ appears in the coefficients of $M_{i}+c N_{i}^{+}-c N_{i}^{-}$since $N_{i}^{+}$and $N_{i}^{-}$have no common components). Further, the coefficient of $A_{i}$ and the sequence $c_{i}$ are increasing. Hence, by Theorem 2.5 , possibly passing to a tail of the sequence, we may assume that $\left(X_{i},\left\lceil A_{i}\right\rceil+M_{i}+c N_{i}^{+}-c N_{i}^{-}\right)$is lc.

Suppose $c_{i}>c$. Then $\left(X_{i}, A_{i}+M_{i}+c N_{i}^{+}-c_{i} N_{i}^{-}\right)$is lc. Here, the coefficients of $M_{i}+c N_{i}^{+}$satisfy the DCC (Lemma 3.13(4)), and the coefficients of $A_{i}$ and the sequence $-c_{i}$ are increasing. Hence, by Theorem 2.5 , possibly passing to a tail of the sequence, we may assume that $\left(X_{i},\left\lceil A_{i}\right\rceil+M_{i}+c N_{i}^{+}-c N_{i}^{-}\right)$is lc.

Set $a_{i}:=\operatorname{mld}\left(X_{i}, \Delta_{i}\left(c_{i}\right)\right) \geq 0$. Possibly passing to a subsequence, it is sufficient to treat the following two cases:

(A) $a_{i}$ is bounded away from zero.

(B) $a_{i}$ approaches zero.

Case B We treat the case where $a_{i}$ approaches zero from above.

Step B-1 We reduce to the case where $A_{i} \neq 0$ and $\left(X_{i}, \Delta_{i}\left(c_{i}\right)\right)$ is dlt.

$\overline{\text { We may }}$ assume that $a_{i} \leq 1$ for any $i$. Take an extraction $\pi_{i}: X_{i}^{\prime} \rightarrow X_{i}$ of a divisor $E_{i}$ computing $\operatorname{mld}\left(\bar{X}_{i}, \Delta_{i}\left(c_{i}\right)\right)=a_{i}$ (Thms. 2.3 and 2.4). We remark that $E_{i}$ may be nonexceptional. Then we may write

$$
K_{X_{i}^{\prime}}+\left(1-a_{i}\right) E_{i}+T_{i}+\Delta_{i}^{\prime}\left(c_{i}\right)=\pi_{i}^{*}\left(K_{X_{i}}+\Delta_{i}\left(c_{i}\right)\right),
$$

where $T_{i}$ is the sum of exceptional divisors (note that $T_{i}=0$ when $a_{i}>0$ ), and $\Delta_{i}^{\prime}(t)$ is the strict transform of $\Delta_{i}(t)$. Then $\left(X_{i}^{\prime},\left(1-a_{i}\right) E_{i}+T_{i}+\Delta_{i}^{\prime}(t)\right)$ satisfies the following conditions:

- We may write $\left(1-a_{i}\right) E_{i}+T_{i}+\Delta_{i}^{\prime}(t)=A_{i}^{\prime}+B_{i}^{\prime}(t)$ with all the conditions in Proposition 3.10.

- $\left\lfloor A_{i}^{\prime}\right\rfloor=\left\lfloor A_{i}^{\prime}+B_{i}^{\prime}\left(c_{i}\right)\right\rfloor$ and $A_{i}^{\prime} \neq 0$.

- $\left(X_{i}^{\prime},\left(1-a_{i}\right) E_{i}+T_{i}+\Delta_{i}^{\prime}\left(c_{i}\right)\right)$ is dlt.

Hence, we may replace $\left(X_{i}, \Delta_{i}(t)\right)$ by $\left(X_{i}^{\prime},\left(1-a_{i}\right) E_{i}+T_{i}+\Delta_{i}^{\prime}(t)\right)$.

Step B-2 We are done if there exists a component $S_{i} \subset \operatorname{Supp}\left\lfloor A_{i}\right\rfloor$ such that $\left.\left(K_{X_{i}}+\Delta_{i}\left(c_{i}^{\prime}\right)\right)\right|_{S_{i}} \neq 0$.

Suppose that there exists a component $S_{i} \subset \operatorname{Supp}\left\lfloor A_{i}\right\rfloor$ such that $\left(K_{X_{i}}+\right.$ $\left.\Delta_{i}\left(c_{i}^{\prime}\right)\right)\left.\right|_{S_{i}} \neq \equiv 0$. By adjunction, we can define $\Delta_{S_{i}}(t)$ as follows:

$$
\left.\left(K_{X_{i}}+\Delta_{i}(t)\right)\right|_{S_{i}}=K_{S_{i}}+\Delta_{S_{i}}(t) .
$$

Then $\left(S_{i}, \Delta_{S_{i}}(t)\right)$ satisfies the following conditions:

- $\operatorname{dim} S_{i} \leq d-1$.

- $K_{S_{i}}+\Delta_{S_{i}}\left(c_{i}^{\prime}\right) \not \equiv 0$ by the assumption.

- $\left(S_{i}, \Delta_{S_{i}}(t)\right)$ satisfies the other conditions in Proposition 3.10. 
Hence, we may replace $\left(X_{i}, \Delta_{i}(t)\right)$ by $\left(S_{i}, \Delta_{S_{i}}(t)\right)$. By induction on $d$ it follows that $c \in \mathfrak{G}_{d-2}(I) \subset \mathfrak{G}_{d-1}(I)$.

Step B-3 We are done if $f_{i}: X_{i} \rightarrow Z_{i}$ is a Mori fiber space with $\operatorname{dim} Z_{i}>0$ and $\overline{\operatorname{Supp} A_{i}}$ dominates $Z_{i}$.

Let $F_{i}$ be the general fiber of $f_{i}$. We may define $\Delta_{F_{i}}(t)$ as follows:

$$
\left.\left(K_{X_{i}}+\Delta_{i}(t)\right)\right|_{F_{i}}=K_{F_{i}}+\Delta_{F_{i}}(t) .
$$

Then $\left(F_{i}, \Delta_{F_{i}}(t)\right)$ satisfies all conditions in Proposition 3.10 except for $K_{F_{i}}+$ $\Delta_{F_{i}}\left(c_{i}^{\prime}\right) \not \equiv 0$. Hence, if $K_{F_{i}}+\Delta_{F_{i}}\left(c_{i}^{\prime}\right) \not \equiv 0$ for some $c_{i}^{\prime}$, then $c \in \mathfrak{G}_{d-2}(I) \subset$ $\mathfrak{G}_{d-1}(I)$ by induction on $d$.

Suppose that $K_{F_{i}}+\Delta_{F_{i}}\left(c_{i}^{\prime}\right) \equiv 0$, and so $K_{F_{i}}+\Delta_{F_{i}}(c) \equiv 0$. We may write $\Delta_{F_{i}}(t)=A_{i}^{\prime}+B_{i}^{\prime}(t)$ with the conditions in Proposition 3.10. Note that $\left(F_{i}, \Delta_{F_{i}}(c)\right)$ is lc by the same reason as Claim 3.14. Since the coefficients of $B_{i}^{\prime}(c)$ satisfies the DCC (Lemma 3.13(4)), it follows that $\left\lfloor A_{i}^{\prime}\right\rfloor=A_{i}^{\prime}$ by Theorem 2.6. Therefore, there exists a component $S_{i} \subset \operatorname{Supp}\left\lfloor A_{i}\right\rfloor$ such that $f\left(S_{i}\right)=Z_{i}$. Since $\left.\left(K_{X_{i}}+\Delta_{i}(c)\right)\right|_{F_{i}} \equiv 0, K_{X_{i}}+\Delta_{i}(c)$ is linearly equivalent to the pulled back of an $\mathbb{R}$-divisor $D_{i}$ on $Z_{i}$. Since $K_{X_{i}}+\Delta_{i}(c) \not \equiv 0$, it follows that $D_{i} \not \equiv 0$, and so $\left.\left(K_{X_{i}}+\Delta_{i}(c)\right)\right|_{S_{i}} \not \equiv 0$. Therefore, we are done by Step B-2.

Step B-4 We finish the case where $\left(X_{i}, \Delta_{i}\left(c_{i}\right)\right)$ is not klt (equivalently, $\left\lfloor A_{i}\right\rfloor \neq$ 0 by Step B-1).

Suppose that $\left(X_{i}, \Delta_{i}\left(c_{i}\right)\right)$ is not klt. Then $\left\lfloor A_{i}\right\rfloor \neq 0$. We run a $\left(K_{X_{i}}+\Delta_{i}\left(c_{i}\right)-\right.$ $\left.\left\lfloor A_{i}\right\rfloor\right)$-MMP. Since $K_{X_{i}}+\Delta_{i}\left(c_{i}\right)-\left\lfloor A_{i}\right\rfloor \equiv-\left\lfloor A_{i}\right\rfloor$ is not pseudo-effective, a $\left(K_{X_{i}}+\Delta_{i}\left(c_{i}\right)-\left\lfloor A_{i}\right\rfloor\right)$-MMP terminates and ends with a Mori fiber space by [4, Cor. 1.3.3].

Let $f_{i}: X_{i} \rightarrow X_{i}^{\prime}$ be a step of the MMP. First, suppose that $f_{i}$ is birational. We write

$$
A_{i}^{\prime}:=f_{i *} A_{i}, \quad B_{i}^{\prime}(t):=f_{i *} B_{i}(t), \quad \Delta_{i}^{\prime}(t)=A_{i}^{\prime}+B_{i}^{\prime}(t) .
$$

Then, $\left(X_{i}^{\prime}, \Delta_{i}^{\prime}(t)\right)$ satisfies all conditions in Proposition 3.10 except for $K_{X_{i}^{\prime}}+$ $\Delta_{i}^{\prime}\left(c_{i}^{\prime}\right) \not \equiv 0$.

Assume that $K_{X_{i}^{\prime}}+\Delta_{i}^{\prime}\left(c_{i}^{\prime}\right) \equiv 0$ (hence, $f_{i}$ is a divisorial contraction). Set $D_{i}:=K_{X_{i}}+\Delta_{i}\left(c_{i}^{\prime}\right) \not \equiv 0$. We may write $D_{i}-f_{i}^{*} f_{i *} D_{i}=a E$, where $E$ is the $f_{i}$-exceptional divisor, and $a \in \mathbb{R}$. Since $D_{i} \not \equiv 0$ and $f_{i *} D_{i} \equiv 0$, we have $a E \not \equiv 0$. Since $f_{i}$ is $\left\lfloor A_{i}\right\rfloor$-positive, there exists a component $T_{i} \subset \operatorname{Supp}\left\lfloor A_{i}\right\rfloor$ such that $\left.E\right|_{T_{i}} \not \equiv 0$. Therefore, we are done by Step B-2.

Hence, we may assume that $K_{X_{i}^{\prime}}+\Delta_{i}^{\prime}\left(c_{i}^{\prime}\right) \not \equiv 0$, replace $\left(X_{i}, \Delta_{i}(t)\right)$ by $\left(X_{i}^{\prime}, \Delta_{i}^{\prime}(t)\right)$, and continue the MMP. The MMP must terminate with a Mori fiber space $f_{i}: X_{i} \rightarrow Z_{i}$. If $\operatorname{dim} Z_{i}=0$, then the Picard number of $X_{i}$ is one. Therefore, $\left.\left(K_{X_{i}}+\Delta_{i}\left(c_{i}^{\prime}\right)\right)\right|_{T_{i}} \not \equiv 0$ for any component $T_{i} \subset \operatorname{Supp}\left\lfloor A_{i}\right\rfloor$, and we are done by Step B-2. Suppose $\operatorname{dim} Z_{i}>0$. Since $f_{i}$ is $\left\lfloor A_{i}\right\rfloor$-positive, $\left\lfloor A_{i}\right\rfloor$ dominates $Z_{i}$, and we are done by Step B-3.

Step B-5 In what follows, we assume that $\left(X_{i}, \Delta\left(c_{i}\right)\right)$ is klt. We reduce to the case where $X_{i}$ has the Picard number one. 
We run a $\left(K_{X_{i}}+B_{i}\left(c_{i}\right)\right)$-MMP. Since $\left(K_{X_{i}}+B_{i}\left(c_{i}\right)\right) \equiv-A_{i}$ is not pseudoeffective, a $\left(K_{X_{i}}+B_{i}\left(c_{i}\right)\right)$-MMP terminates and ends with a Mori fiber space by [4, Cor. 1.3.3].

Let $f_{i}: X_{i} \rightarrow X_{i}^{\prime}$ be a step of the MMP. First, suppose that $f_{i}$ is birational. We write

$$
A_{i}^{\prime}:=f_{i *} A_{i}, \quad B_{i}^{\prime}(t):=f_{i *} B_{i}(t), \quad \Delta_{i}^{\prime}(t)=A_{i}^{\prime}+B_{i}^{\prime}(t) .
$$

Then, $\left(X_{i}^{\prime}, \Delta_{i}^{\prime}(t)\right)$ satisfies all conditions in Proposition 3.10 except for $K_{X_{i}^{\prime}}+$ $\Delta_{i}^{\prime}\left(c_{i}^{\prime}\right) \not \equiv 0$. We prove $K_{X_{i}^{\prime}}+\Delta_{i}^{\prime}\left(c_{i}^{\prime}\right) \not \equiv 0$.

Suppose $K_{X_{i}^{\prime}}+\Delta_{i}^{\prime}\left(c_{i}^{\prime}\right) \equiv 0$. It implies that $K_{X_{i}^{\prime}}+\Delta_{i}^{\prime}(c) \equiv 0$. Note that $\left(X_{i}^{\prime}, \Delta_{i}^{\prime}(c)\right)$ is lc by Claim 3.14. Further, the coefficients of $B_{i}^{\prime}(c)$ satisfy the DCC (Lemma 3.13(4)), the coefficients of $A_{i}$ are approaching 1 , and $\left\lfloor A_{i}\right\rfloor=0$. This contradicts Theorem 2.6.

Since $K_{X_{i}^{\prime}}+\Delta_{i}^{\prime}\left(c_{i}^{\prime}\right) \not \equiv 0$, we may replace $\left(X_{i}, \Delta_{i}(t)\right)$ by $\left(X_{i}^{\prime}, \Delta_{i}^{\prime}(t)\right)$ and continue the MMP. Then the MMP must terminate and ends with a Mori fiber space $X_{i} \rightarrow Z_{i}$. If $\operatorname{dim} Z_{i}=0$, the Picard number of $X_{i}$ is one. Suppose $\operatorname{dim} Z_{i}>0$. Since $f_{i}$ is $A_{i}$-positive, Supp $A_{i}$ dominates $Z_{i}$, and we are done by Step B-3.

Step B-6 We finish case B.

Claim 3.15. We may assume that $K_{X_{i}}+A_{i}+B_{i}(c)$ is not ample for any $i$.

Proof. Assume that $K_{X_{i}}+A_{i}+B_{i}(c)$ is ample. We may write

$$
A_{i}+B_{i}(t)=M_{i}+t\left(N_{i}^{+}-N_{i}^{-}\right) \text {, }
$$

where $N_{i}^{+} \geq 0$ and $N_{i}^{-} \geq 0$ have no common components. Further, we may write

$$
N_{i}^{+} \equiv n_{i}^{+} H_{i}, \quad N_{i}^{-} \equiv n_{i}^{-} H_{i}
$$

with some ample divisor $H_{i}$ and $n_{i}^{+}, n_{i}^{-} \in \mathbb{Q}_{\geq 0}$.

First, suppose $c_{i}>c$. Then $K_{X_{i}}+A_{i}+B_{i}(c) \equiv\left(c-c_{i}\right)\left(N_{i}^{+}-N_{i}^{-}\right)$is ample, and so $n_{i}^{+}<n_{i}^{-}$. Then we have

$$
K_{X_{i}}+M_{i}+c N_{i}^{+}-\left(c_{i}-\left(c_{i}-c\right) \frac{n_{i}^{+}}{n_{i}^{-}}\right) N_{i}^{-} \equiv K_{X_{i}}+M_{i}+c_{i}\left(N_{i}^{+}-N_{i}^{-}\right) \equiv 0 .
$$

Here, we have $c<c_{i}-\left(c_{i}-c\right)\left(n_{i}^{+} / n_{i}^{-}\right)<c_{i}$, and so

$$
0 \leq M_{i}+c N_{i}^{+}-\left(c_{i}-\left(c_{i}-c\right) \frac{n_{i}^{+}}{n_{i}^{-}}\right) N_{i}^{-} \leq M_{i}+c\left(N_{i}^{+}-N_{i}^{-}\right) .
$$

Since $\left(X_{i}, M_{i}+c\left(N_{i}^{+}-N_{i}^{-}\right)\right)$is lc by Claim 3.14, the new pair $\left(X_{i}, M_{i}+c N_{i}^{+}-\right.$ $\left.\left(c_{i}-\left(c_{i}-c\right)\left(n_{i}^{+} / n_{i}^{-}\right)\right) N_{i}^{-}\right)$is also lc, but this contradicts Lemma 3.13(4) and Theorem 2.6.

Suppose $c_{i}<c$. Then we have $n_{i}^{+}>n_{i}^{-}$and

$$
K_{X_{i}}+M_{i}+\left(c_{i}+\left(c-c_{i}\right) \frac{n_{i}^{-}}{n_{i}^{+}}\right) N_{i}^{+}-c N_{i}^{-} \equiv K_{X_{i}}+M_{i}+c_{i}\left(N_{i}^{+}-N_{i}^{-}\right) \equiv 0 .
$$


Here, we have $c_{i}<c_{i}+\left(c-c_{i}\right)\left(n_{i}^{-} / n_{i}^{+}\right)<c$ and

$$
0 \leq M_{i}+\left(c_{i}+\left(c-c_{i}\right) \frac{n_{i}^{-}}{n_{i}^{+}}\right) N_{i}^{+}-c N_{i}^{-} \leq M_{i}+c\left(N_{i}^{+}-N_{i}^{-}\right) .
$$

Note that the first inequality follows from Lemma 3.13(3). Since $\left(X_{i}, M_{i}+\right.$ $\left.c\left(N_{i}^{+}-N_{i}^{-}\right)\right)$is lc by Claim 3.14, the new pair $\left(X_{i}, M_{i}+\left(c_{i}+\left(c-c_{i}\right)\left(n_{i}^{-} /\right.\right.\right.$ $\left.\left.\left.n_{i}^{+}\right)\right) N_{i}^{+}-c N_{i}^{-}\right)$is also lc, but this contradicts Lemma 3.13(4) and Theorem 2.6.

First, suppose that $\left(X_{i},\left\lceil A_{i}\right\rceil+B_{i}\left(c_{i}\right)\right)$ is not lc. Note that $\left(X_{i},\left\lceil A_{i}\right\rceil+B_{i}(c)\right)$ is lc by Claim 3.14. Set

$$
d_{i}:= \begin{cases}\sup \left\{t \in\left[c, c_{i}\right) \mid\left(X_{i},\left\lceil A_{i}\right\rceil+B_{i}(t)\right) \text { is lc }\right\} & \text { when } c<c_{i}, \\ \inf \left\{t \in\left(c_{i}, c\right] \mid\left(X_{i},\left\lceil A_{i}\right\rceil+B_{i}(t)\right) \text { is lc }\right\} & \text { when } c_{i}<c .\end{cases}
$$

Then $d_{i} \in \mathfrak{L}_{d}(I) \subset \mathfrak{G}_{d-1}(I)$, and $\lim d_{i}=\lim c_{i}=c$. Therefore, we are done by induction on $d$.

Thus, we may assume that $\left(X_{i},\left\lceil A_{i}\right\rceil+B_{i}\left(c_{i}\right)\right)$ is lc. Set $e_{i}, f_{i} \in \mathbb{R}$ as

$$
K_{X_{i}}+\left\lceil A_{i}\right\rceil+B_{i}\left(e_{i}\right) \equiv 0, \quad K_{X_{i}}+f_{i}\left\lceil A_{i}\right\rceil+B_{i}(c) \equiv 0 .
$$

Since $B_{i}\left(c_{i}\right)-B_{i}(c)$ is ample (Claim 3.15) and $K_{X_{i}}+A_{i}+B_{i}\left(c_{i}\right) \equiv 0$, there are only two cases:

- $e_{i} \geq c>c_{i}$ or $e_{i} \leq c<c_{i}$, or

- $c \geq e_{i} \geq c_{i}$ or $c \leq e_{i} \leq c_{i}$.

First, suppose that $e_{i} \geq c>c_{i}$ or $e_{i} \leq c<c_{i}$. Then $K_{X_{i}}+\left\lceil A_{i}\right\rceil+B_{i}(c)$ is ample, and so $f_{i}<1$. Further, since $K_{X_{i}}+A_{i}+B_{i}(c)$ is not ample and the coefficients of $A_{i}$ are approaching one, it follows that $\lim f_{i}=1$. Therefore, the set of coefficients of $f_{i}\left\lceil A_{i}\right\rceil+B_{i}(c)$ satisfies the DCC (Lemma 3.13(4)), which contradicts Theorem 2.6.

Next, suppose that $c \geq e_{i} \geq c_{i}$ or $c \leq e_{i} \leq c_{i}$. Thus, we have $\lim e_{i}=$ $\lim c_{i}=c$. Suppose $c \geq e_{i} \geq c_{i}$ (the other case can be proved in the same way). We may assume that $e_{i}<e_{i+1}$ for all $i$ or $e_{i}=c$ for some $i$. In the former case, since the sequence $e_{i}$ is accumulating to $c$, we may replace $\left(X_{i}, \Delta_{i}(t)\right)$ by $\left(X_{i},\left\lceil A_{i}\right\rceil+B_{i}(t)\right)$. Remark that $\left(X_{i},\left\lceil A_{i}\right\rceil+B_{i}\left(e_{i}\right)\right)$ is lc because both $\left(X_{i},\left\lceil A_{i}\right\rceil+B_{i}\left(c_{i}\right)\right)$ and $\left(X_{i},\left\lceil A_{i}\right\rceil+B_{i}(c)\right)$ are lc. Note that the Picard number of $X_{i}$ is one. Hence, for any component of $S_{i} \subset \operatorname{Supp}\left\lceil A_{i}\right\rceil$, we have $\left(K_{X_{i}}+\left\lceil A_{i}\right\rceil+\right.$ $\left.B_{i}\left(c_{i}^{\prime}\right)\right)\left.\right|_{S_{i}} \not \equiv 0$ for some $c_{i}^{\prime}$. Therefore, we are done by Step B-2. In the latter case, $c=e_{i} \in \mathfrak{G}_{d-1}(I)$ by adjunction.

Case A We treat the case where $a_{i}$ is bounded away from zero.

In this case, it follows that $A_{i}=0$ and $\left(X_{i}, B_{i}\left(c_{i}\right)\right)$ is klt.

Step A-1 We reduce to the case where $X_{i}$ has the Picard number one.

$\overline{\text { Since } K_{X_{i}}}+B_{i}\left(c_{i}\right) \equiv 0$ and $K_{X_{i}}+B_{i}\left(c_{i}^{\prime}\right) \not \equiv 0$ for some $c_{i}^{\prime}$, we can take $\epsilon \in \mathbb{R}$ such that $K_{X_{i}}+B_{i}\left(c_{i}+\epsilon\right)$ is klt (Lemma 3.13(2)) and not pseudo-effective. We run a $\left(K_{X_{i}}+B_{i}\left(c_{i}+\epsilon\right)\right)$-MMP. Since $K_{X_{i}}+B_{i}\left(c_{i}+\epsilon\right)$ is not pseudo-effective, a $\left(K_{X_{i}}+B_{i}\left(c_{i}+\epsilon\right)\right)$-MMP terminates and ends with a Mori fiber space [4, Cor. 1.3.3]. 
Let $f_{i}: X_{i} \rightarrow X_{i}^{\prime}$ be a step of the MMP. First, suppose that $f_{i}$ is birational. We write

$$
B_{i}^{\prime}(t):=f_{i *} B_{i}(t), \quad \Delta_{i}^{\prime}(t):=B_{i}^{\prime}(t) .
$$

Then, $\left(X_{i}^{\prime}, \Delta_{i}^{\prime}(t)\right)$ satisfies all conditions in Proposition 3.10 except for $K_{X_{i}^{\prime}}+$ $\Delta_{i}^{\prime}\left(c_{i}^{\prime}\right) \not \equiv 0$. We prove $K_{X_{i}^{\prime}}+\Delta_{i}^{\prime}\left(c_{i}^{\prime}\right) \not \equiv 0$.

Suppose $K_{X_{i}^{\prime}}+\Delta_{i}^{\prime}\left(c_{i}+\epsilon\right) \equiv 0$ (hence, $f_{i}$ is a divisorial contraction). We denote $D:=K_{X_{i}}+\Delta_{i}\left(c_{i}+\epsilon\right)$. Then we may write

$$
D \equiv D-f_{i}^{*} f_{i *} D=a E,
$$

where $E$ is the exceptional divisor, and $a \in \mathbb{R}$. Since $D$ is not pseudo-effective, it follows that $a<0$. This contradicts the fact that $f_{i}$ is $D$-negative.

Since $K_{X_{i}^{\prime}}+\Delta_{i}^{\prime}\left(c_{i}^{\prime}\right) \not \equiv 0$, we may replace $\left(X_{i}, \Delta_{i}(t)\right)$ by $\left(X_{i}^{\prime}, \Delta_{i}^{\prime}(t)\right)$ and continue the MMP. The MMP must terminate with a Mori fiber space $f_{i}: X_{i} \rightarrow Z_{i}$. If $\operatorname{dim} Z_{i}=0$, then the Picard number of $X_{i}$ is one. Suppose $\operatorname{dim} Z_{i}>0$. Let $F_{i}$ be the general fiber of $f_{i}$. Set $\Delta_{F_{i}}(t)$ by adjunction:

$$
\left.\left(K_{X_{i}}+\Delta_{i}(t)\right)\right|_{F_{i}}=K_{F_{i}}+\Delta_{F_{i}}(t) .
$$

Since $f_{i}$ is $\left(K_{X_{i}}+\Delta_{i}\left(c_{i}+\epsilon\right)\right)$-negative, $\left.\left(K_{X_{i}}+\Delta_{i}\left(c_{i}+\epsilon\right)\right)\right|_{F_{i}} \not \equiv 0$. Then $\left(F_{i}, \Delta_{F_{i}}(t)\right)$ satisfies the conditions in Proposition 3.10. Since $\operatorname{dim} F_{i} \leq d-1$, we are done by induction on $d$.

Step A-1' Since $X_{i}$ has the Picard number one, by Lemmas 3.12 and 3.13(2) the numbers of components of $B_{i}(t)$ are bounded. Hence, possibly passing to a subsequence, we may assume that the numbers of components of $B_{i}(t)$ are fixed. Since $a_{i}$ are bounded away from zero, the coefficients of $B_{i}(t)$ have only finitely many possibilities (Lemma 3.13(1)). Therefore, possibly passing to a subsequence, we may assume that the coefficients of $B_{i}(t)$ are fixed and of the form

$$
\frac{m-1+f+k t}{m}
$$

where $m \in \mathbb{Z}_{>0}, f \in I_{+}$, and $k \in \mathbb{Z}$. Here, $m, f$, and $k$ depend on the component but not on $i$.

Set

$$
\begin{aligned}
& h_{i}^{+}:=\sup \left\{t \geq c_{i} \mid\left(X_{i}, B_{i}(t)\right) \text { is } \mathrm{lc}\right\} \geq c_{i}, \\
& h_{i}^{-}:=\inf \left\{t \leq c_{i} \mid\left(X_{i}, B_{i}(t)\right) \text { is } \mathrm{lc}\right\} \leq c_{i} .
\end{aligned}
$$

Since $h_{i}^{+}$and $h_{i}^{-}$are bounded, possibly passing to a subsequence, we may assume that the limits $h^{+}=\lim h_{i}^{+}$and $h^{-}=\lim h_{i}^{-}$exist.

Step A-2 We finish the case where $c \geq h^{+}$or $c \leq h^{-}$.

In this case, we have $h^{+}=\lim h_{i}^{+}=c$ or $h^{-}=\lim h_{i}^{-}=c$. Since $h_{i}^{+}, h_{i}^{-} \in$ $\mathfrak{L}_{d}(I) \subset \mathfrak{G}_{d-1}(I)$, we are done by induction on $d$.

Step A-3 In what follows, we assume that $c<h^{+}$and $c>h^{-}$. Let

$$
d_{i}^{+}=\frac{c_{i}+h_{i}^{+}}{2}, \quad d_{i}^{-}=\frac{c_{i}+h_{i}^{-}}{2}, \quad d^{+}=\frac{c+h^{+}}{2}, \quad d^{-}=\frac{c+h^{-}}{2} .
$$


Then $d^{+}>c$ and $d^{-}<c$. Further, we may assume that $d^{+}>c_{i}$ and $d^{-}<c_{i}$, possibly passing to a tail of the sequence. Note that the following hold:

- If $c_{i}>c$, then $K_{X_{i}}+B_{i}\left(d^{+}\right)$is ample.

- If $c_{i}<c$, then $K_{X_{i}}+B_{i}\left(d^{-}\right)$is ample.

This is because $K_{X_{i}}+B_{i}(c)$ is not ample by the same reason as Claim 3.15.

In this step, we prove that the following hold:

- If $c_{i}>c$, then $\operatorname{vol}\left(X_{i}, K_{X_{i}}+B_{i}\left(d^{+}\right)\right)$is unbounded.

- If $c_{i}<c$, then $\operatorname{vol}\left(X_{i}, K_{X_{i}}+B_{i}\left(d^{-}\right)\right)$is unbounded.

Suppose that $c_{i}>c$ and $\operatorname{vol}\left(X_{i}, K_{X_{i}}+B_{i}\left(d^{+}\right)\right)$is bounded from above (the other case can be proved in the same way). Since the coefficients of $\left(X_{i}, B_{i}\left(d^{+}\right)\right)$ are fixed, there exists $m \in \mathbb{Z}_{>0}$ such that $\phi_{m\left(K_{X_{i}}+B_{i}\left(d^{+}\right)\right)}$is birational for all $i$ by [8, Thm. 1.3]. But then, by [7, Lemma 2.4.2] $\left\{\left(X_{i}, B_{i}\left(d^{+}\right)\right) \mid i \in \mathbb{Z}_{>0}\right\}$ is $\log$ birationally bounded since $\operatorname{vol}\left(X_{i}, K_{X_{i}}+B_{i}\left(d^{+}\right)\right)$is bounded by the assumption. Note that the coefficients of $B_{i}\left(d_{i}^{+}\right)$are bounded from below and $\operatorname{mld}\left(X_{i}, B_{i}\left(d_{i}^{+}\right)\right)$is also bounded from below:

$$
\operatorname{mld}\left(X_{i}, B_{i}\left(d_{i}^{+}\right)\right) \geq \frac{\operatorname{mld}\left(X_{i}, B_{i}\left(h_{i}^{+}\right)\right)+\operatorname{mld}\left(X_{i}, B_{i}\left(c_{i}\right)\right)}{2}=\frac{a_{i}}{2} .
$$

Hence, by [8, Thm. 1.6], $\left\{\left(X_{i}, B_{i}\left(d^{+}\right)\right) \mid i \in \mathbb{Z}_{>0}\right\}$ turns out to be a bounded family.

Thus, we may take an ample Cartier divisor $H_{i}$ on $X_{i}$ such that

$$
T_{i} \cdot H_{i}^{\operatorname{dim} X_{i}-1}, \quad K_{X_{i}} \cdot H_{i}^{\operatorname{dim} X_{i}-1}
$$

are bounded, where $T_{i}$ is any component of $B_{i}(t)$. Hence, we may assume that these intersection numbers are independent of $i$, possibly passing to a subsequence. We may write $B_{i}(t)=M_{i}+t N_{i}$. Since the coefficients of $B_{i}$ are independent of $i$, it follows that $M_{i} \cdot H_{i}^{\operatorname{dim} X_{i}-1}$ and $N_{i} \cdot H_{i}^{\operatorname{dim} X_{i}-1}$ are also constant. Since

$$
0=\left(K_{X_{i}}+B_{i}\left(c_{i}\right)\right) \cdot H_{i}^{\operatorname{dim} X_{i}-1}=\left(K_{X_{i}}+M_{i}+c_{i} N_{i}\right) \cdot H_{i}^{\operatorname{dim} X_{i}-1},
$$

it follows that $c_{i}$ is also constant, a contradiction. Remark that $N_{i} \cdot H_{i}^{\operatorname{dim} X_{i}-1} \neq 0$ since $N_{i} \not \equiv 0$.

Step A-4 By Step A-3 the following hold:

- If $c_{i}>c$, then $K_{X_{i}}+B_{i}\left(d^{+}\right)$is ample, and $\operatorname{vol}\left(X_{i}, K_{X_{i}}+B_{i}\left(d^{+}\right)\right)$is unbounded.

- If $c_{i}<c$, then $K_{X_{i}}+B_{i}\left(d^{-}\right)$is ample, and $\operatorname{vol}\left(X_{i}, K_{X_{i}}+B_{i}\left(d^{-}\right)\right)$is unbounded.

Suppose $c_{i}>c$ (the other case can be proved in the same way). Note that $K_{X_{i}}+B_{i}\left(d^{+}\right) \equiv B_{i}\left(d^{+}\right)-B_{i}\left(c_{i}\right)$. Then, by Lemmas 3.2.2 and 3.2.3 in [8], possibly passing to a tail of the sequence, we may find $g_{i}<c_{i}$ and an $\mathbb{R}$-divisor $\Theta_{i}$ with the following conditions:

- $0 \leq \Theta_{i} \sim_{\mathbb{R}} B_{i}\left(c_{i}\right)-B_{i}\left(g_{i}\right)$.

- $B_{i}\left(g_{i}\right) \geq 0$ (cf. Lemma 3.13(3)). 
- $\lim g_{i}=c$.

- $\left(X_{i}, B_{i}\left(g_{i}\right)+\Theta_{i}\right)$ has a unique non-klt place.

Let $\phi: Y_{i} \rightarrow X_{i}$ be a dlt modification of $\left(X_{i}, B_{i}\left(g_{i}\right)+\Theta_{i}\right)$. Then we may write

$$
K_{Y_{i}}+B_{i}^{\prime}\left(g_{i}\right)+\Theta_{i}^{\prime}+S_{i}=\phi^{*}\left(K_{X_{i}}+B_{i}\left(g_{i}\right)+\Theta_{i}\right),
$$

where $S_{i}$ is the unique exceptional divisor, and $B_{i}^{\prime}(t)$ and $\Theta_{i}^{\prime}$ are the strict transforms of $B_{i}(t)$ and $\Theta_{i}$. We may also write

$$
K_{Y_{i}}+B_{i}^{\prime}\left(c_{i}\right)+s_{i} S_{i}=\phi^{*}\left(K_{X_{i}}+B_{i}\left(c_{i}\right)\right)
$$

with $s_{i}<1$ since $\left(X_{i}, B_{i}\left(c_{i}\right)\right)$ is klt.

Claim 3.16. We may assume that $S_{i}$ is ample and $K_{Y_{i}}+B_{i}^{\prime}\left(l_{i}\right)+S_{i} \equiv 0$ for some $l_{i} \in\left[g_{i}, c_{i}\right)$.

First, we assume this claim and finish the proof.

Suppose that $\left(Y_{i}, B_{i}^{\prime}\left(c_{i}\right)+S_{i}\right)$ is not lc. Note that $\left(Y_{i}, B_{i}^{\prime}\left(g_{i}\right)+S_{i}\right)$ is lc. Set

$$
k_{i}:=\sup \left\{t \in\left[g_{i}, c_{i}\right) \mid\left(Y_{i}, B_{i}^{\prime}(t)+S_{i}\right) \text { is lc }\right\} .
$$

Then $k_{i} \in \mathfrak{L}_{d}(I) \subset \mathfrak{G}_{d-1}(I)$, and $\lim k_{i}=c$. Therefore, we are done by induction on $d$.

Thus, we may assume that $\left(Y_{i}, B_{i}^{\prime}\left(c_{i}\right)+S_{i}\right)$ is lc. By adjunction, we can define $B_{i}^{\prime \prime}(t)$ as follows:

$$
\left.\left(K_{Y_{i}}+B_{i}^{\prime}(t)+S_{i}\right)\right|_{S_{i}}=K_{S_{i}}+B_{i}^{\prime \prime}(t) .
$$

Since $\left(Y_{i}, B_{i}^{\prime}\left(c_{i}\right)+S_{i}\right)$ is lc, it follows that $B_{i}^{\prime \prime}(t) \in \mathcal{D}_{c_{i}}(I)$ by Lemma 3.2. Further, $\left(S_{i}, B_{i}^{\prime \prime}\left(c_{i}\right)\right)$ and $\left(S_{i}, B_{i}^{\prime \prime}\left(g_{i}\right)\right)$ are lc. By Claim 3.16 it follows that $K_{S_{i}}+B_{i}^{\prime \prime}\left(l_{i}\right) \equiv$ 0 and $K_{S_{i}}+B_{i}^{\prime \prime}\left(c_{i}\right) \not \equiv 0$. Therefore, $l_{i} \in \mathfrak{G}_{d-1}(I)$. Since $\lim l_{i}=c$, we are done by induction on $d$.

Proof of Claim 3.16. We run a $\left(K_{Y_{i}}+B_{i}^{\prime}\left(g_{i}\right)+\Theta_{i}^{\prime}\right)$-MMP. Since $\left(Y_{i}, B_{i}^{\prime}\left(g_{i}\right)+\right.$ $\left.\Theta_{i}^{\prime}\right)$ is klt and $K_{Y_{i}}+B_{i}^{\prime}\left(g_{i}\right)+\Theta_{i}^{\prime} \equiv-S_{i}$ is not pseudo-effective, a $\left(K_{Y_{i}}+B_{i}^{\prime}\left(g_{i}\right)+\right.$ $\Theta_{i}^{\prime}$ )-MMP $f_{i}: Y_{i} \rightarrow W_{i}$ terminates and ends with a Mori fiber space $\pi_{i}: W_{i} \rightarrow$ $Z_{i}$ by [4, Cor. 1.3.3].

Let $F_{i}$ be the general fiber of $\pi_{i}$, and let $B_{i}^{\prime \prime \prime}(t), \Theta_{i}^{\prime \prime \prime}$, and $S_{i}^{\prime \prime \prime}$ be the restrictions of $f_{i *} B_{i}^{\prime}(t), f_{i *} \Theta_{i}^{\prime}$, and $f_{i *} S_{i}$ to $F_{i}$. Note that $S_{i}^{\prime \prime \prime} \neq 0$ since every step of this MMP is $S_{i}$-positive. Further, $B_{i}^{\prime \prime \prime}(t), \Theta_{i}^{\prime \prime \prime}$, and $S_{i}^{\prime \prime \prime}$ are multiples of the same ample divisor. Therefore, $S_{i}^{\prime \prime \prime}$ is ample. Since

$$
K_{F_{i}}+B_{i}^{\prime \prime \prime}\left(g_{i}\right)+\Theta_{i}^{\prime \prime \prime}+S_{i}^{\prime \prime \prime} \equiv 0, \quad \text { and } \quad K_{F_{i}}+B_{i}^{\prime \prime \prime}\left(c_{i}\right)+s_{i} S_{i}^{\prime \prime \prime} \equiv 0,
$$

we may find

$$
K_{F_{i}}+B_{i}^{\prime \prime \prime}\left(l_{i}\right)+S_{i}^{\prime \prime \prime} \equiv 0
$$

for some $l_{i} \in\left[g_{i}, c_{i}\right)$. Therefore, we can apply the same argument before after replacing $\left(Y_{i}, B_{i}^{\prime}(t)+S_{i}\right)$ by $\left(F_{i}, B_{i}^{\prime \prime \prime}(t)+S_{i}^{\prime \prime \prime}\right)$. 
Proof of Corollary 3.9. By Theorems 3.6 and 3.8, it is sufficient to show that $\mathfrak{G}_{d}(I) \subset \operatorname{Span}_{\mathbb{Q}}(I \cup\{1\})$ for any $d$.

Let $c \in \mathfrak{G}_{d}(I)$. Then, by the definition of $\mathfrak{G}_{d}(I)$ (Def. 3.5) there exist a $\mathbb{Q}$ factorial normal projective variety $X$ and an affine functional divisor $\Delta(t) \in$ $\mathcal{D}_{c}(I)$ such that $K_{X}+\Delta(c) \equiv 0$ and $K_{X}+\Delta\left(c^{\prime}\right) \not \equiv 0$ for some $c^{\prime} \neq c$. Here, we can take a curve $C$ satisfying $\left(K_{X}+\Delta\left(c^{\prime}\right)\right) \cdot C \neq 0$. Since $\Delta(t) \in \mathcal{D}_{c}(I)$, we may write $\left(K_{X}+\Delta(t)\right) \cdot C=\alpha t+\beta$ with $\alpha \in \mathbb{Q}$ and $\beta \in \operatorname{Span}_{\mathbb{Q}}(I \cup\{1\})$. Since $\alpha c+\beta=0$, we have $c=-\beta \alpha^{-1} \in \operatorname{Span}_{\mathbb{Q}}(I \cup\{1\}$ ) (note that $\alpha \neq 0$ since $\left.\alpha c^{\prime}+\beta \neq 0\right)$.

\section{Perturbation of Irrational Coefficients of LC Pairs}

The goal of this section is to prove Theorem 1.6. The ideal setting is treated as Theorem 4.1.

Proof of Theorem 1.6. We may write the $\mathbb{Q}$-linear functions $s_{i}$ as

$$
s_{i}\left(x_{0}, \ldots, x_{c^{\prime}}\right)=\sum_{0 \leq j \leq c^{\prime}} q_{i j} x_{j}
$$

with $q_{i j} \in \mathbb{Q}$. Since $s_{i}\left(r_{0}, \ldots, r_{c^{\prime}}\right) \in \mathbb{R}_{\geq 0}$ and $r_{0}, \ldots, r_{c^{\prime}}$ are $\mathbb{Q}$-linearly independent, we can take $t^{-}, t^{+} \in \mathbb{Q}$ with the following conditions:

- $t^{-}<r_{c^{\prime}}<t^{+}$, and

- $s_{i}\left(r_{0}, \ldots, r_{c^{\prime}-1}, t\right) \in \mathbb{R}_{\geq 0}$ for any $t$ satisfying $t^{-} \leq t \leq t^{+}$.

Suppose that the statement does not hold. Then there exist $\mathbb{Q}$-Gorenstein varieties $X^{(l)}\left(l \in \mathbb{Z}_{>0}\right)$ of dimension $d$ and $\mathbb{Q}$-Cartier effective Weil divisors $D_{0}^{(l)}, \ldots, D_{c}^{(l)}$ on $X^{(l)}$ such that the following hold:

- $\left(X^{(l)}, \sum_{1 \leq i \leq c} s_{i}\left(r_{0}, \ldots, r_{c^{\prime}}\right) D_{i}^{(l)}\right)$ is lc, and

- $\lim h_{l}^{+}=r_{c^{\prime}}$ or $\lim h_{l}^{-}=r_{c^{\prime}}$,

where we set

$$
\begin{aligned}
& h_{l}^{+}:=\sup \left\{t \geq r_{c^{\prime}} \mid\left(X^{(l)}, \sum_{1 \leq i \leq c} s_{i}\left(r_{0}, \ldots, r_{c^{\prime}-1}, t\right) D_{i}^{(l)}\right) \text { is lc }\right\}, \\
& h_{l}^{-}:=\inf \left\{t \leq r_{c^{\prime}} \mid\left(X^{(l)}, \sum_{1 \leq i \leq c} s_{i}\left(r_{0}, \ldots, r_{c^{\prime}-1}, t\right) D_{i}^{(l)}\right) \text { is lc }\right\} .
\end{aligned}
$$

Suppose that $\lim h_{l}^{-}=r_{c^{\prime}}$ (the other case can be proved in the same way). We may assume that $t^{-} \leq h_{l}^{-} \leq r_{c^{\prime}}$. Note that

$$
\begin{aligned}
\sum_{1 \leq i \leq c} s_{i}\left(r_{0}, \ldots, r_{c^{\prime}-1}, t\right) D_{i}^{(l)}= & \sum_{1 \leq i \leq c} s_{i}\left(r_{0}, \ldots, r_{c^{\prime}-1}, t^{-}\right) D_{i}^{(l)} \\
& +\left(t-t^{-}\right) \sum_{1 \leq i \leq c} q_{i c^{\prime}} D_{i}^{(l)} .
\end{aligned}
$$

Let

$$
I:=\left\{s_{i}\left(r_{0}, \ldots, r_{c^{\prime}-1}, t^{-}\right) \mid 1 \leq i \leq c\right\} .
$$


This becomes a finite set. Take $m \in \mathbb{Z}_{>0}$ such that $m q_{i c^{\prime}} \in \mathbb{Z}$ for any $i$. Then $\left(h_{l}^{-}-t^{-}\right) / m \in \mathfrak{L}_{d}(I)$. Hence, by Corollary 3.9 it follows that

$$
\frac{r_{c^{\prime}}-t^{-}}{m} \in \operatorname{Span}_{\mathbb{Q}}(I \cup\{1\}) \subset \operatorname{Span}_{\mathbb{Q}}\left(r_{0}, \ldots, r_{c^{\prime}-1}\right) .
$$

This contradicts the $\mathbb{Q}$-linear independence of $r_{0}, \ldots, r_{c^{\prime}}$.

The case of the pair with ideal sheaves can be also proved.

TheOREM 4.1. Fix $d \in \mathbb{Z}_{>0}$. Let $r_{1}, \ldots, r_{c^{\prime}}$ be positive real numbers, and let $r_{0}=1$. Assume that $r_{0}, \ldots, r_{c^{\prime}}$ are $\mathbb{Q}$-linearly independent. Let $s_{1}, \ldots, s_{c}$ : $\mathbb{R}^{c^{\prime}+1} \rightarrow \mathbb{R}$ be $\mathbb{Q}$-linear functions from $\mathbb{R}^{c^{\prime}+1}$ to $\mathbb{R}$. Assume that $s_{i}\left(r_{0}, \ldots, r_{c^{\prime}}\right) \in$ $\mathbb{R}_{\geq 0}$ for each $i$. Then there exists a positive real number $\epsilon>0$ with the following conditions:

- $s_{i}\left(r_{0}, \ldots, r_{c^{\prime}-1}, t\right) \geq 0$ for any $t$ satisfying $\left|t-r_{c^{\prime}}\right| \leq \epsilon$.

- For any $\mathbb{Q}$-Gorenstein normal variety $X$ of dimension d and any ideal sheaves $\mathfrak{a}_{1}, \ldots, \mathfrak{a}_{c}$ on $X$, if the pair $\left(X, \prod_{1 \leq i \leq c} \mathfrak{a}_{i}^{s_{i}\left(r_{0}, \ldots, r_{c^{\prime}}\right)}\right)$ is lc, then the pair $\left(X, \prod_{1 \leq i \leq c} \mathfrak{a}_{i}^{s_{i}\left(r_{0}, \ldots, r_{c^{\prime}-1}, t\right)}\right)$ is also lc for any $t$ satisfying $\left|t-r_{c^{\prime}}\right| \leq \epsilon$.

This theorem follows from Theorem 1.6 by the following lemma (cf. [17, Prop. 9.2.28]).

Lemma 4.2. Fix $l \in \mathbb{Z}_{>0}$. Let $X$ be a $\mathbb{Q}$-Gorenstein normal affine variety, and let $\mathfrak{a}_{1}, \ldots, \mathfrak{a}_{c}$ be ideal sheaves on $X$. Fix general elements $f_{i 1}, \ldots, f_{i l} \in \mathfrak{a}_{i}$ for each $i$, and let $D_{i j}=\operatorname{div}\left(f_{i j}\right) \geq 0$ be the corresponding Cartier divisors. Set $D_{i}:=\sum_{1 \leq j \leq l} D_{i j}$.

Then the following holds for any positive real numbers $r_{1}, \ldots, r_{c} \leq l$ at most $l$ : the pair $\left(X, \prod_{1 \leq i \leq c} \mathfrak{a}_{i}^{r_{i}}\right)$ is lc if and only if the pair $\left(X, \frac{1}{l} \sum_{1 \leq i \leq c} r_{i} D_{i}\right)$ is lc.

Definition 4.3. Let $X$ be an affine variety, and $\mathfrak{a}$ an ideal sheaf. Fix generators $g_{1}, \ldots, g_{c} \in \mathfrak{a}$. Then, a general element of $\mathfrak{a}$ is a general $\mathbb{C}$-linear combination of $g_{i}$.

Proof of Lemma 4.2. Let $\mathfrak{b}_{i}$ be an ideal sheaf generated by $\prod_{1 \leq j \leq l} f_{i j}$. Then the pair $\left(X, \frac{1}{l} \sum_{1 \leq i \leq c} r_{i} D_{i}\right)$ is corresponding to the pair $\left(X, \prod_{1 \leq i \leq c} \mathfrak{b}_{i}^{r_{i} / l}\right)$.

Since $\mathfrak{b}_{i} \subset \mathfrak{a}_{i}^{l}$, it easily follows that the log canonicity of $\left(X, \prod_{1 \leq i \leq c} \mathfrak{b}_{i}^{r_{i} / l}\right)$ implies the log canonicity of $\left(X, \prod_{1 \leq i \leq c} \mathfrak{a}_{i}^{r_{i}}\right)$.

Suppose that $\left(X, \prod_{1 \leq i \leq c} \mathfrak{a}_{i}^{r_{i}}\right)$ is lc. Let $Y \rightarrow X$ be a $\log$ resolution of $\left(X, \prod_{1 \leq i \leq c} \mathfrak{a}_{i}^{r_{i}}\right)$. Then we may write $\mathfrak{a}_{i} \mathcal{O}_{Y}=\mathcal{O}_{Y}\left(-E_{i}\right)$ with some Cartier divisor $E_{i}$. Since $\mathfrak{b}_{i} \subset \mathfrak{a}_{i}^{l}$, we may write $\mathfrak{b}_{i} \mathcal{O}_{Y}=\mathfrak{c}_{i} \mathcal{O}_{Y}\left(-l E_{i}\right)$ with some ideal sheaf $\mathfrak{c}_{i} \subset \mathcal{O}_{Y}$. Let $e_{i}$ be a local generator of $\mathcal{O}_{Y}\left(-E_{i}\right)$. Then $\mathfrak{c}_{i}$ is generated by $\prod_{1 \leq j \leq l} g_{i j}$, where we set $g_{i j}:=f_{i j} e_{i}^{-1} \in \mathcal{O}_{Y}$. Since $f_{i 1}, \ldots, f_{i l}$ are general elements of $\mathfrak{a}_{i}$, the elements $g_{i 1}, \ldots, g_{i l}$ become general elements of $\mathcal{O}_{Y}$. Therefore, $Y \rightarrow X$ is also a $\log$ resolution of $\left(X, \prod_{1 \leq i \leq c} \mathfrak{b}_{i}^{r_{i} / l}\right)$. Since $\operatorname{ord}_{g_{i j}} \mathfrak{b}_{i}^{r_{i} / l}=r_{i} / l \leq 1$, it follows that $\left(X, \prod_{1 \leq i \leq c} \mathfrak{b}_{i}^{r_{i} / l}\right)$ is also lc. 


\section{Proof of Main Theorem and Corollaries}

Theorem 1.2 can be proved by the induction on $\operatorname{dim}_{\mathbb{Q}} \operatorname{Span}_{\mathbb{Q}}(I \cup\{1\})$. The same argument essentially appears in [10].

Proof of Theorem 1.2. It is sufficient to prove the case where $1 \in I$. Let $r_{0}=1$, $r_{1}, \ldots, r_{c}$ be all the elements of $I$. Set $c^{\prime}+1:=\operatorname{dim}_{\mathbb{Q}} \operatorname{Span}_{\mathbb{Q}}\left(1, r_{1}, \ldots, r_{c}\right)$. Possibly rearranging the indices, we may assume that $r_{0}, \ldots, r_{c^{\prime}}$ are $\mathbb{Q}$-linearly independent. We may write $r_{i}=\sum_{0 \leq j \leq c^{\prime}} q_{i j} r_{j}$ with $q_{i j} \in \mathbb{Q}$.

We prove by induction on $c^{\prime}$. If $c^{\prime}=0$, then we can take $n \in \mathbb{Z}_{>0}$ such that $I \subset \frac{1}{n} \mathbb{Z}$ and $\frac{1}{r} \in \frac{1}{n} \mathbb{Z}$. Then $B(d, r, I) \subset \frac{1}{n} \mathbb{Z}$, and $B(d, r, I)$ turns out to be discrete.

Set $\mathbb{Q}$-linear functions $s_{0}, \ldots, s_{c}$ as follows:

$$
s_{i}: \mathbb{R}^{c^{\prime}+1} \rightarrow \mathbb{R} ; \quad s_{i}\left(x_{0}, \ldots, x_{c^{\prime}}\right)=\sum_{0 \leq j \leq c^{\prime}} q_{i j} x_{j} .
$$

Take $\epsilon>0$ as in Theorem 4.1. We fix $t^{+}, t^{-} \in \mathbb{Q}$ such that

$$
t^{+} \in\left(r_{c^{\prime}}, r_{c^{\prime}}+\epsilon\right] \cap \mathbb{Q}, \quad t^{-} \in\left[r_{c^{\prime}}-\epsilon, r_{c^{\prime}}\right) \cap \mathbb{Q} .
$$

We define $r_{0}^{+}, \ldots, r_{c}^{+}$and $r_{0}^{-}, \ldots, r_{c}^{-}$as

$$
r_{i}^{+}=s_{i}\left(r_{0}, \ldots, r_{c^{\prime}-1}, t^{+}\right), \quad r_{i}^{-}=s_{i}\left(r_{0}, \ldots, r_{c^{\prime}-1}, t^{-}\right) .
$$

Further, we set $I^{\prime}:=\left\{r_{0}^{+}, \ldots, r_{c}^{+}, r_{0}^{-}, \ldots, r_{c}^{-}\right\}$. Then $\operatorname{dim}_{\mathbb{Q}} \operatorname{Span}_{\mathbb{Q}}\left(I^{\prime}\right)=c^{\prime}$, and so $B\left(d, r, I^{\prime}\right)$ is discrete by induction.

Let $\left(X, \prod_{0 \leq i \leq c} \mathfrak{a}_{i}^{r_{i}}\right) \in P(d, r)$, and let $E$ be a divisor over $X$. Since $(X$, $\left.\prod_{0 \leq i \leq c} \mathfrak{a}_{i}^{r_{i}}\right)$ is lc, $\left(X, \prod_{0 \leq i \leq c} \mathfrak{a}_{i}^{r_{i}^{*}}\right)$ is also lc for each $* \in\{+,-\}$. Hence, we have

$$
\begin{aligned}
0 & \leq a_{E}\left(X, \prod_{0 \leq i \leq c} \mathfrak{a}_{i}^{r_{i}^{*}}\right) \\
& =a_{E}\left(X, \prod_{0 \leq i \leq c} \mathfrak{a}_{i}^{r_{i}}\right)-\left(r_{c^{\prime}}^{*}-r_{c^{\prime}}\right) \sum_{0 \leq i \leq c} q_{i c^{\prime}} \operatorname{ord}_{E} \mathfrak{a}_{i} .
\end{aligned}
$$

Therefore, either of the following holds:

- $0 \leq \sum_{0 \leq i \leq c} q_{i c^{\prime}} \operatorname{ord}_{E} \mathfrak{a}_{i} \leq \epsilon_{+}^{-1} a_{E}\left(X, \prod_{0 \leq i \leq c} \mathfrak{a}_{i}^{r_{i}}\right)$, or

- $-\epsilon_{-}^{-1} a_{E}\left(X, \prod_{0 \leq i \leq c} \mathfrak{a}_{i}^{r_{i}}\right) \leq \sum_{0 \leq i \leq c} q_{i c^{\prime}} \operatorname{ord}_{E} \mathfrak{a}_{i} \leq 0$,

where we set $\epsilon_{+}:=r_{c^{\prime}}^{+}-r_{c^{\prime}}$ and $\epsilon_{-}:=r_{c^{\prime}}-r_{c^{\prime}}^{-}$.

It is sufficient to show the discreteness of $B(d, r, I) \cap[0, a]$ for any $a \in \mathbb{R}_{>0}$. Take $n \in \mathbb{Z}_{>0}$ such that $q_{i c^{\prime}} \in \frac{1}{n} \mathbb{Z}$ for any $i$. Then, it is sufficient to prove that $B(d, r, I) \cap[0, a]$ is contained in

$$
\begin{aligned}
\left\{b+\epsilon_{+} e \mid b \in B\left(d, r, I^{\prime}\right), e \in \frac{1}{n} \mathbb{Z} \cap\left[0, \epsilon_{+}^{-1} a\right]\right\} \\
\cup\left\{b-\epsilon_{-} e \mid b \in B\left(d, r, I^{\prime}\right), e \in \frac{1}{n} \mathbb{Z} \cap\left[-\epsilon_{-}^{-1} a, 0\right]\right\} .
\end{aligned}
$$

In fact, this set becomes discrete because $B\left(d, r, I^{\prime}\right)$ is discrete, and both $\frac{1}{n} \mathbb{Z} \cap$ $\left[0, \epsilon_{+}^{-1} a\right]$ and $\frac{1}{n} \mathbb{Z} \cap\left[-\epsilon_{-}^{-1} a, 0\right]$ are finite. 
Let $\left(X, \prod_{0 \leq i \leq c} \mathfrak{a}_{i}^{r_{i}}\right) \in P(d, t)$, and $E$ a divisor over $X$. Assume that $a_{E}(X$, $\left.\prod_{0 \leq i \leq c} \mathfrak{a}_{i}^{r_{i}}\right) \in[0, a]$. Further, suppose $\sum_{0 \leq i \leq c} q_{i c^{\prime}} \operatorname{ord}_{E} \mathfrak{a}_{i} \geq 0$ (the other case can be proved in the same way). Then, we have

$$
a_{E}\left(X, \prod_{0 \leq i \leq c} \mathfrak{a}_{i}^{r_{i}}\right)=a_{E}\left(X, \prod_{0 \leq i \leq c} \mathfrak{a}_{i}^{r_{i}^{+}}\right)+\left(r_{c^{\prime}}^{+}-r_{c^{\prime}}\right) \sum_{0 \leq i \leq c} q_{i c^{\prime}} \operatorname{ord}_{E} \mathfrak{a}_{i} .
$$

Here, we have

- $a_{E}\left(X, \prod_{0 \leq i \leq c} \mathfrak{a}_{i}^{r_{i}^{+}}\right) \in B\left(d, r, I^{\prime}\right)$,

- $r_{c^{\prime}}^{+}-r_{c^{\prime}}=\epsilon_{+}$, and

- $\sum_{0 \leq i \leq c} q_{i c^{\prime}} \operatorname{ord}_{E} \mathfrak{a}_{i} \in \frac{1}{n} \mathbb{Z} \cap\left[0, \epsilon_{+}^{-1} a\right]$.

We complete the proof.

Proof of Corollary 1.5. Note that $A_{\mathrm{can}}(3, I) \subset[1,3]$ (cf. [13; 18]). We prove that for any $a>1$, the set

$$
A_{\text {can }}(3, I) \cap[a,+\infty)
$$

is a finite set.

By the classification of three-dimensional $\mathbb{Q}$-factorial terminal singularities (see $[13 ; 18])$ the minimal log discrepancy of a three-dimensional terminal singularity is equal to $1+1 / r\left(r \in \mathbb{Z}_{>0}\right)$ or 3 . In the case where $\operatorname{mld}_{x}(X)=3$, the Gorenstein index of $X$ at $x$ is 1 . If $\operatorname{mld}_{x}(X)=1+1 / r$, then the Gorenstein index of $X$ at $x$ is $r$. Further, by [12, Cor. 5.2], if $X$ has Gorenstein index $r$ at $x \in X$, then $r D$ is Cartier at $x$ for any Weil divisor $D$.

Let $(X, \Delta)$ be a three-dimensional canonical pair satisfying $\Delta \in I$ and $\operatorname{mld}_{x}(X, \Delta) \geq a$. By [4, Cor. 1.4.3], there exists a projective morphism $f: Y \rightarrow X$ with the following properties:

- $Y$ is a $\mathbb{Q}$-factorial terminal variety.

- $f^{*}\left(K_{X}+\Delta\right)=K_{Y}+\Delta_{Y}$, where $\Delta_{Y}$ is the strict transform on $Y$ of $\Delta$ (note that $(X, \Delta)$ is canonical).

Take a divisor $E$ over $X$ such that $\operatorname{mld}_{x}(X, \Delta)=a_{E}(X, \Delta)$ and $\mathrm{c}_{X}(E)=\{x\}$.

Suppose $\operatorname{dimc}_{Y}(E)=0$. Then $\operatorname{mld}_{x}(X, \Delta)=\operatorname{mld}_{y}\left(Y, \Delta_{Y}\right)$, where $\{y\}:=$ $\mathrm{c}_{Y}(E)$. Since $\operatorname{mld}_{y}(Y) \geq \operatorname{mld}_{y}\left(Y, \Delta_{Y}\right) \geq a$, the Gorenstein index of $Y$ at $y$ is at most $\left\lfloor\frac{1}{a-1}\right\rfloor$. Let $l$ be the Gorenstein index of $Y$ at $y$. Since $l D$ is Cartier at $y$ for any Weil divisor $D$ on $Y$, it follows that $\operatorname{mld}_{y}\left(Y, \Delta_{Y}\right) \in A^{\prime}\left(3, l, \frac{1}{l} I\right)$ (see Remark 2.2), where we set

$$
\frac{1}{l} I:=\left\{f l^{-1} \mid f \in I\right\}
$$

Therefore, we have

$$
\operatorname{mld}_{x}(X, \Delta) \in \bigcup_{l \leq\lfloor 1 /(a-1)\rfloor} A^{\prime}\left(3, l, \frac{1}{l} I\right),
$$

and the right-hand side is a finite set by Corollary 1.3.

Suppose $\operatorname{dimc}_{Y}(E)=1$. Then, by [2, Prop. 2.1],

$$
\operatorname{mld}_{y}\left(Y, \Delta_{Y}\right)=1+\operatorname{mld}_{x}(X, \Delta)
$$


for some $y \in \mathrm{c}_{Y}(E)$. Since $\operatorname{mld}_{y}(Y) \geq 1+a>2$, it follows that $Y$ has Gorenstein index 1 . Hence,

$$
\operatorname{mld}_{y}\left(Y, \Delta_{Y}\right) \in A^{\prime}(3,1, I) .
$$

Therefore, we have

$$
\operatorname{mld}_{x}(X, \Delta) \in-1+A^{\prime}(3,1, I),
$$

and the right-hand side is a finite set by Corollary 1.3.

Suppose $\operatorname{dim}_{Y}(E)=2$. Then $E$ is a divisor on $Y$, and we have

$$
\operatorname{mld}_{x}(X, \Delta)=1-\operatorname{coeff}_{E} \Delta_{Y} \text {. }
$$

Therefore, we have

$$
\operatorname{mld}_{x}(X, \Delta) \in 1-I,
$$

and the right-hand side is a finite set.

ACKNOWLEDGments. The author expresses his gratitude to his advisor Professor Yujiro Kawamata for his encouragement and valuable advice. He is grateful to Professors Yoshinori Gongyo, Masayuki Kawakita, Shunsuke Takagi, and Hiromu Tanaka for useful comments and suggestions. He is supported by the Grantin-Aid for Scientific Research (KAKENHI No. 25-3003) and the Program for Leading Graduate Schools, MEXT, Japan.

\section{References}

[1] V. Alexeev, Two two-dimensional terminations, Duke Math. J. 69 (1993), no. 3, 527 545.

[2] F. Ambro, On minimal log discrepancies, Math. Res. Lett. 6 (1999), no. 5-6, 573580.

[3] _ The set of toric minimal log discrepancies, Cent. Eur. J. Math. 4 (2006), no. 3, 358-370, (electronic).

[4] C. Birkar, P. Cascini, C. D. Hacon, and J. McKernan, Existence of minimal models for varieties of log general type, J. Amer. Math. Soc. 23 (2010), no. 2, 405-468.

[5] T. de Fernex and M. Mustaţă, Limits of log canonical thresholds, Ann. Sci. Éc. Norm. Supér. (4) 42 (2009), no. 3, 491-515.

[6] O. Fujino, Fundamental theorems for the log minimal model program, Publ. Res. Inst. Math. Sci. 47 (2011), no. 3, 727-789.

[7] C. D. Hacon, J. McKernan, and C. Xu, On the birational automorphisms of varieties of general type, Ann. of Math. (2) 177 (2013), no. 3, 1077-1111.

[8] _ ACC for log canonical thresholds, Ann. of Math. (2) 180 (2014), no. 2, $523-571$.

[9] M. Kawakita, Towards boundedness of minimal log discrepancies by the RiemannRoch theorem, Amer. J. Math. 133 (2011), no. 5, 1299-1311.

[10] _ Discreteness of log discrepancies over log canonical triples on a fixed pair, arXiv: $1204.5248 \mathrm{v} 1$.

[11] _ A connectedness theorem over the spectrum of a formal power series ring, arXiv: $1403.7582 \mathrm{v} 1$.

[12] Y. Kawamata, Crepant blowing-up of 3-dimensional canonical singularities and its application to degenerations of surfaces, Ann. of Math. (2) 127 (1988), no. 1, 93163. 
[13] The minimal discrepancy coefficients of terminal singularities in dimension 3, Appendix to V. V. Shokurov, Three-dimensional log perestroikas, Izv. Ross. Akad. Nauk Ser. Mat. 56 (1992), no. 1, 105-203.

[14] J. Kollár, Which powers of holomorphic functions are integrable? arXiv:0805.0756v1.

[15] J. Kollár and S. Mori, Birational geometry of algebraic varieties, Cambridge Tracts in Math., 134, Cambridge University Press, Cambridge, 1998.

[16] J. Kollár, et al., Flips and abundance for algebraic threefolds, Papers from the second summer seminar on algebraic geometry held at the University of Utah, Salt Lake City, Utah, August 1991, Astérisque, 211, Société Mathématique de France, Paris, 1992.

[17] R. Lazarsfeld, Positivity in algebraic geometry. II, Ergeb. Math. Grenzgeb. (3), 49, Springer-Verlag, Berlin, 2004.

[18] D. Markushevich, Minimal discrepancy for a terminal cDV singularity is 1, J. Math. Sci. Univ. Tokyo 3 (1996), no. 2, 445-456.

[19] J. McKernan and Y. Prokhorov, Threefold thresholds, Manuscripta Math. 114 (2004), no. 3, 281-304.

[20] V. V. Shokurov, A. c. c. in codimension 2, preprint, 1993.

[21] _ 3-Fold log models, J. Math. Sci. 81 (1996), no. 3, 2667-2699, Algebraic geometry 4.

[22] Letters of a bi-rationalist. V. Minimal log discrepancies and termination of log flips, Tr. Mat. Inst. Steklova 246 (2004), 328-351, English transl., Proc. Steklov Inst. Math. 3 (246) (2004), 315-336.

Graduate School of Mathematical

Sciences

University of Tokyo

3-8-1 Komaba

Meguro-ku

Tokyo 153-8914

Japan

nakamura@ms.u-tokyo.ac.jp 